\title{
Bronchial thermoplasty decreases airway remodelling by blocking epithelium- derived heat shock protein-60 secretion and protein arginine methyltransferase-1 in fibroblasts
}

\author{
Qingzhu Sun ${ }^{1,2,6}$, Lei Fang ${ }^{2,6}$, Michael Roth ${ }^{2}$, Xuemei Tang ${ }^{1}$, \\ Eleni Papakonstantinou ${ }^{2}$, Weiqi Zhai ${ }^{1}$, Renaud Louis ${ }^{3}$, Vincent Heinen ${ }^{3}$, \\ Florence N. Schleich ${ }^{3}$, Shemin $\mathrm{Lu}^{4}$, Spasenjia Savic ${ }^{5}$, Michael Tamm ${ }^{2}$ and \\ Daiana Stolz ${ }^{2}$
}

@ERSpublications

Bronchial thermoplasty reduces asthma-associated airway wall remodelling by epithelial cell-controlled downregulation of PRMT1 expression in fibroblasts http://bit.ly/2TLhCkC

Cite this article as: Sun Q, Fang L, Roth M, et al. Bronchial thermoplasty decreases airway remodelling by blocking epithelium-derived heat shock protein-60 secretion and protein arginine methyltransferase- 1 in fibroblasts. Eur Respir J 2019; 54: 1900300 [https://doi.org/10.1183/13993003.00300-2019].

ABSTRACT Bronchial thermoplasty (BT) is to date the only therapy that provides a lasting reduction in airway wall remodelling. However, the mechanism of action of BT is not well understood. This study aimed to characterise the changes of remodelling regulating signalling pathways by BT in asthma.

Bronchoalveolar lavage fluid (BALF) was obtained from eight patients with severe asthma before and after BT. Primary bronchial epithelial cells were isolated from 23 patients before $(n=66)$ and after $(n=62)$ BT. Epithelial cell culture supernatant (Epi.S) was collected and applied to primary fibroblasts.

Epithelial cells obtained from asthma patients after BT proliferated significantly faster compared with epithelial cells obtained before BT. In airway fibroblasts, BALF or Epi.S obtained before BT increased CCAAT enhancer-binding protein- $\beta(\mathrm{C} / \mathrm{EBP} \beta)$ expression, thereby downregulating microRNA-19a. This upregulated extracellular signal-regulated kinase-1/2 (ERK1/2) expression, protein arginine methyltransferase-1 (PRMT1) expression, cell proliferation and mitochondrial mass. BALF or Epi.S obtained after BT reduced the expression of C/EBP $\beta$, ERK1/2, peroxisome proliferator-activated receptor- $\gamma$ coactivator-1 $\alpha$ (PGC1 $\alpha)$, PRMT1 and mitochondrial mass in airway fibroblasts. Proteome and transcriptome analyses indicated that epithelial cell-derived heat shock protein-60 (HSP60) is the main mediator of BT effects on fibroblasts. Further analysis suggested that HSP60 regulated PRMT1 expression, which was responsible for the increased mitochondrial mass and $\alpha$-smooth muscle actin expression by asthmatic fibroblasts. These effects were ablated after BT. These results imply that BT reduces fibroblast remodelling through modifying the function of epithelial cells, especially by reducing HSP60 secretion and subsequent signalling pathways that regulate PRMT1 expression.

We therefore hypothesise that BT decreases airway remodelling by blocking epithelium-derived HSP60 secretion and PRMT1 in fibroblasts.

This article has been revised according to the correction published in the July 2021 issue of the European Respiratory Journal.

This article has supplementary material available from erj.ersjournals.com

Received: 16 May 2018 | Accepted after revision: 13 Aug 2019

Copyright @ERS 2019 


\section{Introduction}

Asthma is characterised by irreversible structural changes of the airway wall $[1,2]$, resulting in hyperresponsiveness and stiffness of the tissue [3,4]. Remodelling is not reduced by asthma therapies, except bronchial thermoplasty (BT) $[5,6]$. In clinical trials, BT improved quality of life, and reduced exacerbation rates and hospitalisation, which correlated with reduced airway remodelling $[6,7]$.

The function of the airways is regulated by the interaction of epithelial and subepithelial mesenchymal cells. In asthma, this communication is disrupted, leading to irreversible tissue structural changes $[8,9]$. Airway wall remodelling is resistant to all available asthma drugs [10, 11], whereas BT was found to modify the function of subepithelial mesenchymal cells and the composition of the extracellular matrix, and to reduce inflammation $[5,6,12,13]$. BT was also found to reduce the mass of airway smooth muscle $[5,6,14]$, and decrease neuroendocrine-activated epithelial cells and inflammation [11-13]. However, it is unclear if all these effects resulted from "burning off smooth muscle".

Thus, BT might achieve its beneficial effects by modulating the methylation of DNA, histone-3 or noncoding RNA, which have been linked to asthma-associated remodelling $[15,16]$. A major enzyme controlling methylation is protein arginine methyltransferase-1 (PRMT1), which was constitutively upregulated in airway smooth muscle cells of asthma patients [17]. PRMT1 contributes to the pathogenesis of lung cancer, pulmonary fibrosis, pulmonary hypertension, chronic obstructive pulmonary disease (COPD) and asthma [18]. In an animal model of allergic asthma, PRMT1 was cell type specifically regulated in airway epithelial and mesenchymal cells during acute and chronic inflammation [19-22]. Platelet-derived growth factor-BB stimulated PRMT1 expression and remodelling by downregulating microRNA-19a (miR-19a), thereby upregulating extracellular signal-regulated kinase-1/2 (ERK1/2) mitogen-activated protein kinase (MAPK) expression and activity [17, 23].

This study investigated if BT affects the regulatory mechanism of PRMT1 on mitochondria activity and subsequent remodelling in airway fibroblasts.

\section{Methods}

\section{Patient cohorts}

In total, 66 endobronchial tissue biopsies were obtained from 51 patients with severe asthma (according to Global Initiative for Asthma guidelines) by the lung clinic (University Hospital Basel, Basel, Switzerland) before BT and 62 biopsies were obtained from 23 patients at 4,8 and 12 weeks after BT. BT was performed according to a standardised protocol. Table 1 presents the clinical characteristics of the patients.

All patients provided written informed consent. The study was approved by the local Institutional Ethical Committee of Northwest and Central Switzerland (EKNZ 2016-01057).

\section{Study design}

Endobronchial biopsies and bronchoalveolar lavage fluid (BALF) were collected before and after BT. Tissue biopsies were used to isolate primary epithelial cells and fibroblasts (see the Cell isolation and characterisation section). BALF was aliquoted and used for proteomic analysis or was stored $\left(-20^{\circ} \mathrm{C}\right)$ for experiments with fibroblasts. A graphic summary of the study design is provided in figure 1.

\section{BALF and bronchoscopy}

BALF was collected and bronchoscopy was performed according to standard procedures before and after BT.

\section{BALF proteomic analysis and Kyoto Encyclopedia of Genes and Genomes pathway analysis}

Proteomic analyses were performed in BALF samples of seven asthma patients before and after BT and compared in pairs. Mass spectrometric analysis was performed in $200 \mu \mathrm{L}$ BALF by the SWATH method on a Q-Exactive HF platform (Thermo Fisher Scientific, Waltham, MA, USA) and analysed by Spectronaut software (https://biognosys.com) against the Proteomics Core Facility internal HEK cell line spectral library (Biocentre, University of Basel, Basel, Switzerland). The retention time was calibrated by internal indexed retention time standard peptides (iRT Kit; Biognosys). All data are provided as supplementary material.

Affiliations: ${ }^{1}$ College of Animal Science and Technology, Northwest A\&F University, Yangling, China. ${ }^{2}$ Pneumology and Pulmonary Cell Research, Depts of Internal Medicine and Biomedicine, University of Basel and University Hospital Basel, Basel, Switzerland. ${ }^{3}$ Dept of Pneumology, University of Liege, Liege, Belgium. ${ }^{4}$ Dept of Biochemistry and Molecular Biology, Xi'an Jiaotong University Health Science Centre, Xi'an, China. ${ }^{5}$ Dept of Pathology, University Hospital Basel, Basel, Switzerland. ${ }^{6}$ These authors contributed equally to this work.

Correspondence: Michael Roth, Pulmonary Cell Research, Dept of Biomedicine, University of Basel and University Hospital Basel, Petersgraben 4, 4031 Basel, Switzerland. E-mail: Michael.Rothdusb.ch 
TABLE 1 Clinical characteristics of the patient cohorts before and after bronchial thermoplasty (BT)

\begin{tabular}{|c|c|c|c|}
\hline & Before BT & After BT & p-value \\
\hline Subjects & 52 & 25 & \\
\hline \multicolumn{4}{|l|}{ Sex } \\
\hline Female & 26 & 13 & \\
\hline Male & 26 & 12 & \\
\hline Age years & $56.9 \pm 1.4$ & $61.6 \pm 2.5$ & $0.046^{\#}$ \\
\hline $\mathrm{BMI} \mathbf{k g} \cdot \mathrm{m}^{-2}$ & $25.9 \pm 0.5$ & $27.5 \pm 1.1$ & $0.29^{\#}$ \\
\hline Atopy & 30 & 8 & $0.77^{\Uparrow}$ \\
\hline Pre-BD FEV $1 \%$ pred & $65.1 \pm 2.2$ & $63.0 \pm 2.8$ & $0.63^{\#}$ \\
\hline Post-BD FEV $1 \%$ pred & $71.8 \pm 2.3$ & $68.2 \pm 2.7$ & $0.44^{\#}$ \\
\hline Post-BD FEV 1 /FVC \% & $58.4 \pm 1.6$ & $52.1 \pm 1.7$ & $0.041^{\#}$ \\
\hline TLC \% pred & $102.8 \pm 1.9$ & $103.0 \pm 2.7$ & $0.96^{\#}$ \\
\hline RV \% pred & $112.4 \pm 2.1$ & $105.3 \pm 3.8$ & $0.23^{\#}$ \\
\hline$c D_{\text {Lco }} \%$ pred & $88.1 \pm 2.1$ & $90.1 \pm 3.0$ & $0.66^{\#}$ \\
\hline$F_{\text {ENO }}$ & $46.2 \pm 3.9$ & $34.3 \pm 4.1$ & $0.14^{\#}$ \\
\hline ICS/LABA & 45 & 19 & $0.66^{\text {7 }}$ \\
\hline LAMA & 20 & 12 & $0.02^{q}$ \\
\hline OCS & 23 & 13 & $0.07^{\Uparrow}$ \\
\hline Current or previous omalizumab use & 3 & 0 & NA \\
\hline
\end{tabular}

Data are presented as $\mathrm{n}$ or mean $\pm \mathrm{SD}$, unless otherwise stated. BMI: body mass index; BD: bronchodilator; $\mathrm{FEV}_{1}$ : forced expiratory volume in $1 \mathrm{~s}$; FVC: forced vital capacity; TLC: total lung capacity; RV: residual volume; $\mathrm{c} D$ LCO: diffusing capacity of the lung for carbon monoxide corrected for haemoglobin level; $F_{\mathrm{ENO}}$ : exhaled nitric oxide fraction; ICS: inhaled corticosteroid; LABA: long-acting $\beta$-agonist; LAMA: long-acting muscarinic antagonist; OCS: oral corticosteroid; NA: not applicable. " : t-test; ": Chi-squared four fields statistic.

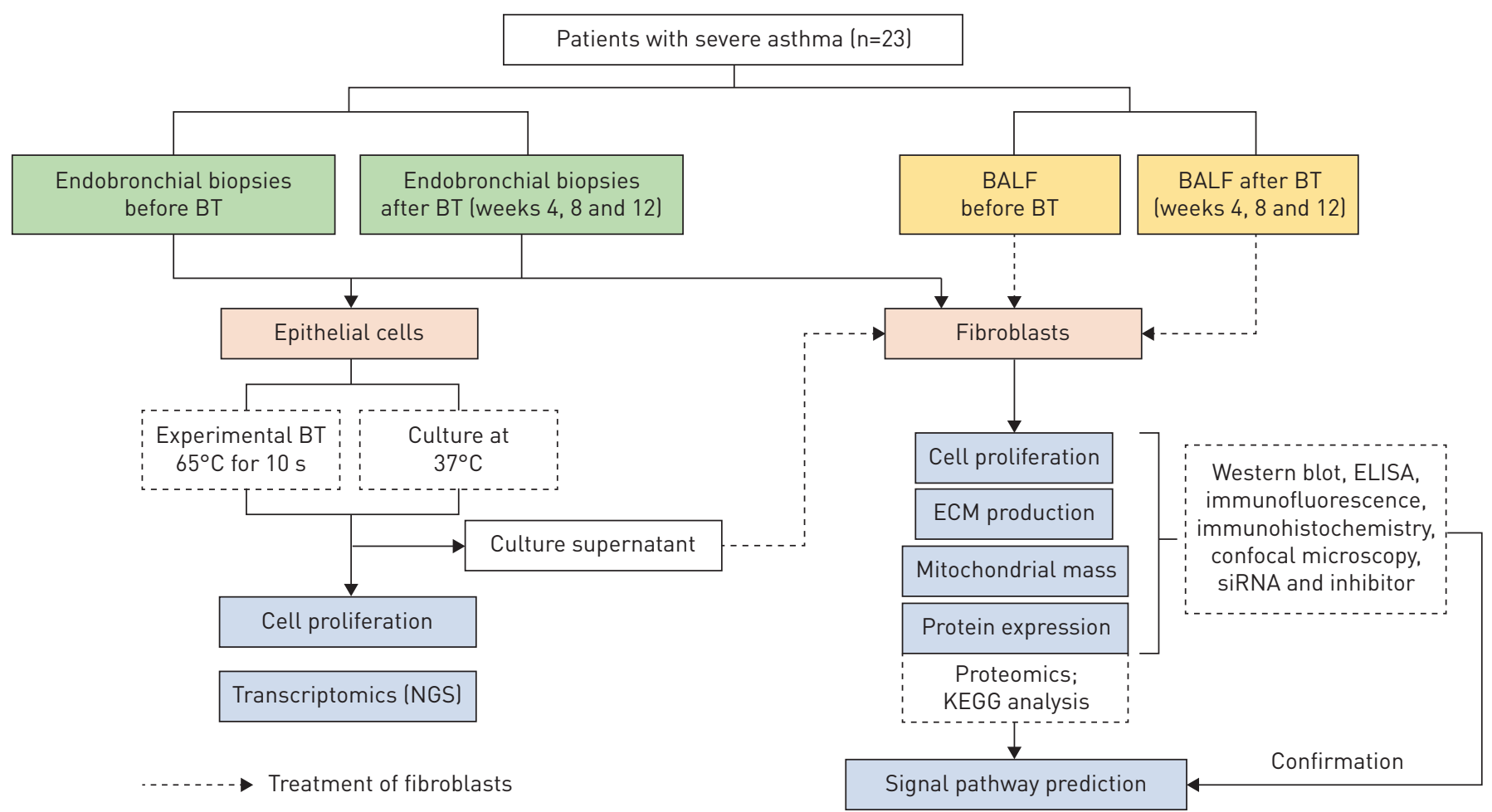

FIGURE 1 Study design. BT: bronchial thermoplasty; BALF: bronchoalveolar lavage fluid; ECM: extracellular matrix; siRNA: small interfering RNA; NGS: next-generation sequencing; KEGG: Kyoto Encyclopedia of Genes and Genomes. Endobronchial biopsies and BALF were obtained from 23 patients with severe asthma before and after undergoing BT. Primary epithelial cells and fibroblasts were isolated by selective medium from each biopsy. Experimental BT $\left(10 \mathrm{~s}, 65^{\circ} \mathrm{C}\right.$ submerged in a water bath) was performed with epithelial cells, and cell culture medium was collected after $24 \mathrm{~h}$ and used to treat fibroblasts. In parallel, fibroblasts were exposed to BALF (diluted 1:1 in fibroblast medium) over different time intervals. 
Statistical analysis of BALF proteomics was performed using OmicShare tools (www.omicshare.com/tools) after removing results with low abundance values. Kyoto Encyclopedia of Genes and Genomes (KEGG) pathway analysis was conducted to identify BT-modified signalling pathways by comparing protein profiles before and after BT.

\section{Cell isolation and characterisation}

Primary bronchial epithelial cells were isolated from biopsies by cell type selective medium CnT-PR-A (CellnTec, Bern, Switzerland), and were characterised by positive staining for E-cadherin and cytokeratin-13 and negative staining for fibronectin (supplementary figure S1). The culture epithelial cell supernatant (Epi.S) was collected from confluent epithelial cells.

Subepithelial bronchial fibroblasts were generated from nonasthma $(n=8)$ and asthma $(n=10)$ patients in selective medium (CnT-PR-F; CellnTec), and were characterised by positive staining for fibronectin and negative staining for E-cadherin and fibrilar $\alpha$-smooth muscle actin ( $\alpha$-SMA) (supplementary figure S1) [20]. All experiments were performed between passage 2 and 5 .

\section{Next-generation sequencing}

Next-generation sequencing (NGS) analysis was performed in primary airway epithelial cell lines $(n=9)$ obtained before and after experimental BT. Confluent cell layers $(25 \mathrm{~cm}$ flasks) were exposed for $10 \mathrm{~s}$ to either $37^{\circ} \mathrm{C}$ (control) or $65^{\circ} \mathrm{C}$ (experimental BT) in a water bath. The flasks were then placed in a humidified incubator for $24 \mathrm{~h}$ at $37^{\circ} \mathrm{C}, 5 \% \mathrm{CO}_{2}$. Total RNA was isolated and assessed for differential mRNA expression of the entire human genome by NGS. RNA libraries were prepared using a TruSeq Stranded mRNA Sample Kit (Illumina, San Diego, CA, USA). mRNA was enriched from total RNA (500 ng) by an oligo-dT bead system and fragmented enzymatically. First- and second-strand syntheses were performed, and double-stranded cDNA was purified (AMPure XP; Thermo Fisher Scientific). The cDNA was $3^{\prime}$-adenylated and Illumina sequencing adaptors ligated onto the fragments. mRNA libraries were pre-amplified by PCR and purified (AMPure XP), and the size distribution was validated and quality inspected on a BioAnalyzer 4200 TapeStation (Agilent Technologies, Santa Clara, CA, USA). High-quality libraries were pooled at equimolar concentrations (BioAnalyzer Smear Analysis; Agilent Technologies) and quantified by quantitative PCR. Optimised library pools were used to generate clusters on the surface of a flow cell before sequencing on a NextSeq 500 instrument (75 cycles; Illumina). The NGS analysis report is provided as supplementary material.

\section{Fibroblast stimulation and small interfering RNA treatment}

Fibroblasts grown in six-well plates were serum deprived $(0.1 \%$ serum $)$ overnight before being treated with Epi.S or BALF (mixed 1:1 with basic medium) for 24 and $48 \mathrm{~h}$.

PRMT1 activity was inhibited by $2 \mathrm{~h}$ pre-incubation with $10 \mu \mathrm{M}$ of the protein arginine methyltransferase inhibitor AMI-1 (Santa Cruz Biotechnology, Dallas, TX, USA).

Quiescent fibroblasts were treated with heat shock protein-60 (HSP60) (1 $\mu \mathrm{g} \cdot \mathrm{mL}^{-1}$; Sino Biological, Beijing, China) or transfected by HiPerFect (TaKaRa, Kusatsu, Japan) for $48 \mathrm{~h}$ with CCAAT enhancer-binding protein- $\beta$ (C/EBP $\beta$ ) small interfering RNAs (siRNAs) or control sequences (all $50 \mathrm{nM}$ ) [19-23].

\section{miR-19a real-time quantitative PCR}

miR-19a was detected in $500 \mathrm{ng}$ of total RNA as described previously $[17,23]$ using the primer sequence 5'-TGT GCA AAT CTA TGC AAA ACT GA-3'. miR-19a expression was normalised to U6 small nuclear RNA and analysed by the $2^{-\Delta \Delta C t}$ (relative quantification) method [17].

\section{Western blotting}

Fibroblasts were lysed in RIPA buffer and protein concentration was quantified by bicinchoninic acid (Thermo Fisher Scientific). Equal amounts of denatured proteins $(20 \mu \mathrm{g})$ were size fractionated by electrophoresis (8-16\% SDS-PAGE; Thermo Fisher Scientific) and transferred onto polyvinylidene fluoride membranes. Proteins were detected by antibodies (PRMT1, peroxisome proliferator-activated receptor- $\gamma$ coactivator-1 $\alpha(\mathrm{PGC1} \alpha)$ or HSP60 (Abcam, Cambridge, UK), or phosphorylated ERK1/2 MAPK, total ERK1/2 MAPK, C/EBP $\beta$ or glyceraldehyde 3-phosphate dehydrogenase (Cell Signaling Technology, Danvers, MA, USA)). Protein bands were visualised after binding of species-specific secondary horseradish peroxidase-conjugated antibodies (Abcam) by chemiluminescence (Thermo Fisher Scientific) [19-23].

\section{Immunofluorescence}

Fibroblasts, seeded on $10 \mathrm{~mm}$ sterile glass slides and serum deprived overnight, were treated with Epi.S or BALF for $0-48 \mathrm{~h}$, fixed in $4 \%$ paraformaldehyde (in PBS, $2 \times 5 \mathrm{~min}$ ) and immunofluorescence staining 
was performed as described previously [17, 23]. Nuclei were stained by 4',6-diamidino-2-phenylindole (DAPI).

\section{MitoTracker staining}

MitoTracker (Thermo Fisher Scientific) staining was performed in quiescent fibroblasts (80\% confluence) as described previously [23]. MitoTracker imaging was captured by an Olympus BX61 fluorescence microscope (Olympus Optical, Tokyo, Japan). Nuclei were stained by DAPI. Image analysis was performed by ImageJ version 2.0 (https://imagej.nih.gov/ij).

\section{Fibroblast activity and proliferation}

Fibroblast activity was determined after incubation $\left(4 \mathrm{~h}, \quad 37^{\circ} \mathrm{C}\right)$ with $500 \mu \mathrm{g} \cdot \mathrm{mL}^{-1}$ MTT (3-(4,5-dimethylthiazol-2-yl)-2,5-diphenyltetrazolium bromide), before being lysed with dimethyl sulfoxide and absorbance measured at $560 \mathrm{~nm}$ (microplate reader; BioTek, Winooski, VT, USA).

Manual cell counts (Neubauer chamber slide) were performed after $48 \mathrm{~h}$ and in the presence or absence of chemical inhibitors for signalling proteins or PRMT1 (AMI-1) [17, 23].

Epithelial cell numbers were determined by calculating the number of cells within a circle of $0.5 \mathrm{~cm}$ diameter every second day over 10 days.

\section{ELISA of collagen type I and fibronectin deposition}

Deposition of collagen type I and fibronectin was measured by ELISA [23]. Confluent fibroblasts in 96-well plates were starved for $24 \mathrm{~h}$ and stimulated with Epi.S. Collagen type I and fibronectin were detected after $24 \mathrm{~h}$ (both antibodies 1:200; Santa Cruz Biotechnology).

\section{Immunohistochemistry}

Bronchial biopsies were embedded in paraffin, cut into $5 \mu \mathrm{m}$ slices and fixed onto glass slides. After de-paraffinisation, rehydration and antigen unmasking by standard procedures, slides were blocked in $5 \%$ bovine serum albumin (BSA), stained for PRMT1 and phosphorylated ERK1/2 MAPK $\left(4^{\circ} \mathrm{C}\right.$, overnight), followed by antibody detection [19-23]. Images were examined by an Olympus BX61 white balance microscope. The percentage of positive cells was determined by double-blind visual quantification.

\section{Confocal microscopy}

Fibroblasts seeded onto coverslips were incubated according to experiments, followed by fixation and cell permeabilisation $[17,23]$. Coverslips were blocked by $5 \%$ BSA in PBS, and then incubated with anti-cytochrome $c$ antibody (BD Biosciences, San Jose, CA, USA) and Alexa Fluor 488-labelled secondary antibody (Thermo Fisher Scientific). F-actin was stained with TRIC-phalloidin and nuclei were stained with DAPI. Images were acquired by a Nikon Confocal A1 microscope ( $\times 401.3 \mathrm{NA}$ FI oil objective of $0.225 \mu \mathrm{m}$ Z-stack step; Nikon, Tokyo, Japan). Images are presented as Z-stack projections by imaging software FIJI (https://imagej.net/Fiji). Single-cell cytochrome $c$ expression was calculated by FIJI with the “3D objects counter" plugin.

\section{Statistical analysis}

The null hypothesis was that no treatment had any effect on signalling or PRMT1 expression. All data are expressed as mean \pm SEM. The statistical analysis was performed by ANOVA followed by the t-test for paired sample data. The effect of inhibitors on PRMT1 and remodelling were analysed by one-way ANOVA. p-values $<0.05$ were regarded as significant.

\section{Results}

BT altered the function of bronchial epithelial cells

The generation of primary bronchial epithelial cells from tissues obtained after BT was significantly faster compared with cells grown from tissue obtained before BT. The increase of cells was determined by direct cell counts over 10 days (figure $2 \mathrm{a}$ and $\mathrm{b}$ ).

In addition to growing in direct contact to the biopsy tissue, epithelial cells growing at a distance $>1 \mathrm{~cm}$ were defined as a "colony". Before BT, 92\% of all epithelial cells grew directly from the biopsy and expanded by forming a continuous cell monolayer. Thus, proliferation occurred mainly on the front of the epithelial cell layer (figure 2c). After BT, epithelial cells were significantly more often observed to grow at a distance from the biopsy, as soon as 1 day after placing the tissue in a flask (figure $2 \mathrm{~d}$ ). The position of such single epithelial cells was marked on the culture dish and cell numbers were counted daily. Within 2 days, single cells multiplied locally forming a "colony" by definition (figure 2e). Within 1 week, the colonies formed a homogenous monolayer of epithelial cells. 

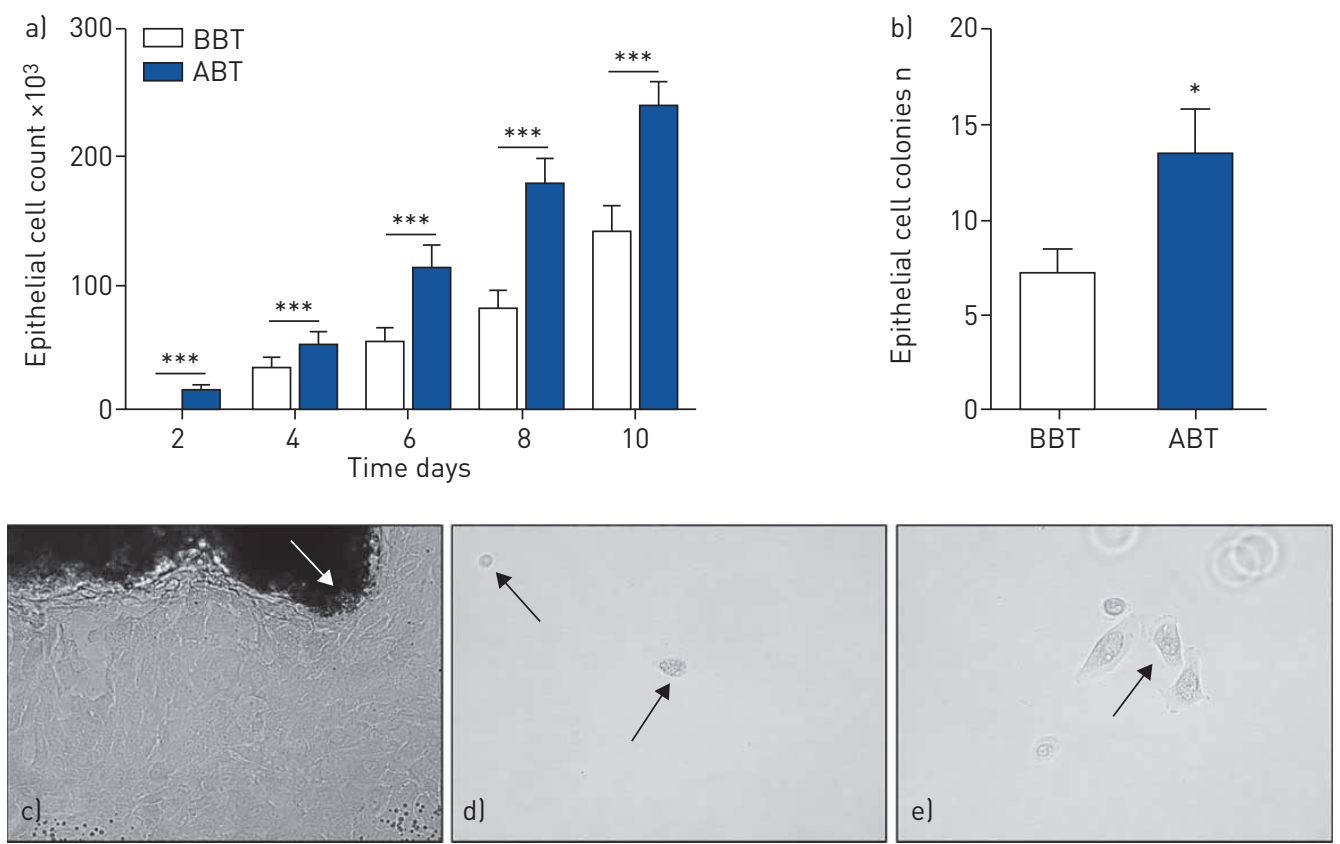

FIGURE 2 Bronchial thermoplasty (BT) altered the function of bronchial epithelial cells. BBT: before BT; ABT: after BT. a) Proliferation of epithelial cells isolated ABT was increased compared with epithelial cells isolated from the same patients BBT ( $n=18)$. b) The motility of epithelial cells increased ABT as monitored by counting epithelial cell colonies at a distance $>1 \mathrm{~cm}$ from the biopsy $(n=12)$. Data are presented as mean \pm SEM. *: $p<0.05$; ***: $p<0.001$, comparing BBT versus ABT. c) Representative microscopic image of epithelial cell growth out from a tissue biopsy obtained BBT. d) Single epithelial cell at a distance $>1 \mathrm{~cm}$ from tissue on the first day after starting the cell cultures of biopsies obtained ABT. e) Same single epithelial cell as shown in (d) 2 days later, forming a colony.

\section{BT reduced ERK1/2 MAPK activation, PRMT1 expression and mitochondrial mass in lung tissue and primary fibroblasts}

In tissue sections of asthma patients obtained before BT, ERK1/2 MAPK was phosphorylated in most epithelial cells and in the subepithelial mesenchymal cells. The ERK1/2 MAPK phosphorylation was significantly reduced in tissues obtained after BT (figure 3a). PRMT1 was mainly located in the nuclei (figure 3a; dark brown/black) and only a few cells showed cytosolic expression (figure 3a; brown), and both were reduced after BT, even 2 years after BT (supplementary figure S2). In isolated fibroblasts, BALF obtained before BT significantly decreased the expression of miR-19a, while BALF obtained after BT did not have this effect (figure 3b). As shown by representative Western blots and image analysis, BALF obtained before BT significantly increased ERK1/2 MAPK phosphorylation, and C/EBP $\beta$ and PRMT1 expression, while BALF obtained after BT did not (figure 3c).

Significantly increased mitochondrial mass in fibroblasts as monitored by cytochrome $c$ expression was observed in BALF obtained before BT (figure 3d; middle row) compared with BALF obtained after BT (figure 3d; bottom row). No difference was found comparing the mitochondrial mass in nonasthma patients $(n=5)$ with asthma patients $(n=6)$. Thus, the data were combined for image analysis, which is presented in figure $3 \mathrm{~d}$ as a bar chart.

Similar to BALF obtained before BT, Epi.S obtained from epithelial cells before BT stimulated PRMT1 expression by fibroblasts significantly when compared with Epi.S obtained from epithelial cells after BT (figure 4). Epi.S obtained after BT had a reduced stimulating effect on PRMT1 expression in fibroblasts. The effect on PRMT1 expression was time dependent and maximal after $48 \mathrm{~h}$, but did not show a disease-specific effect (figure 4).

$B T$ reduced the stimulatory effect of Epi.S on fibroblast proliferation and mitochondrial mass Epi.S of epithelial cells obtained before BT induced ERK1/2 MAPK phosphorylation, and PRMT1 and PGC1 $\alpha$ expression. Western blot data was summarised as a bar chart (figure $5 \mathrm{a}$ ) and confirmed by immunochemistry (figure $5 \mathrm{~b}$ and $\mathrm{c}$ ). The expression levels of PRMT1 and PGC1 $\alpha$ correlated with MitoTracker signals (figure $5 \mathrm{~d}$ ). 
a)
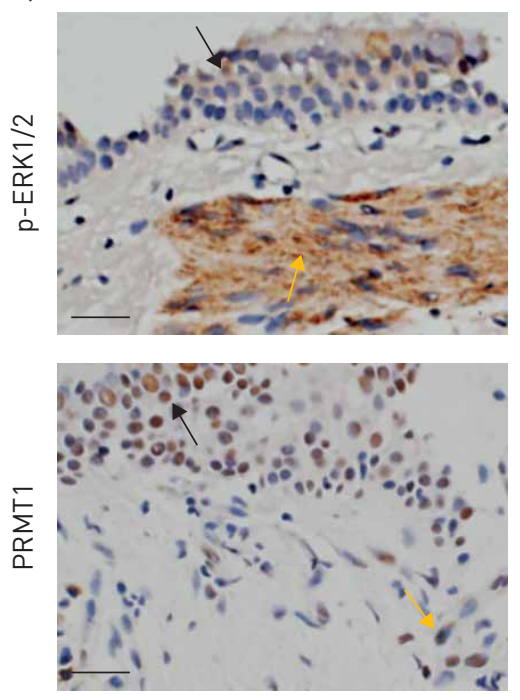

$\mathrm{ABT}$
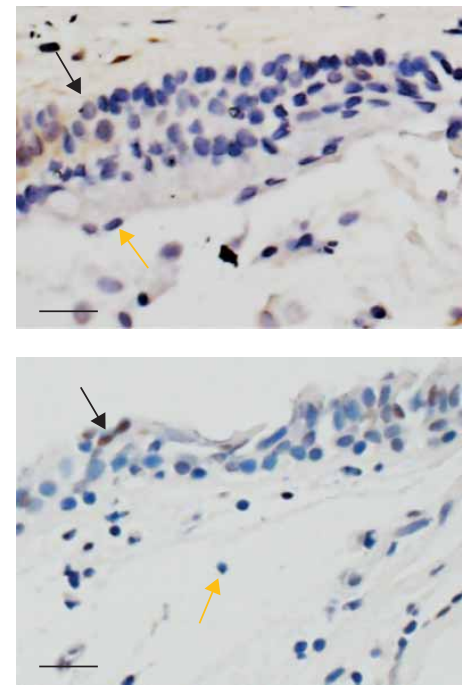
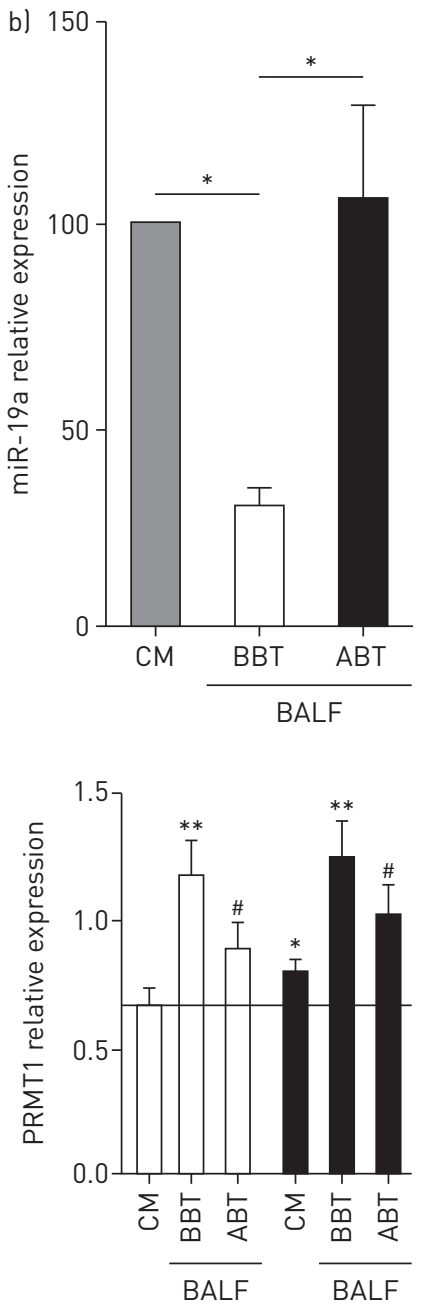

c)

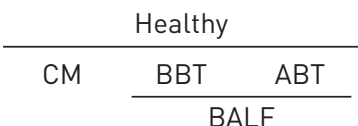

C/EBP $\beta$

p-ERK1/2

t-ERK1/2

PRMT1

GAPDH
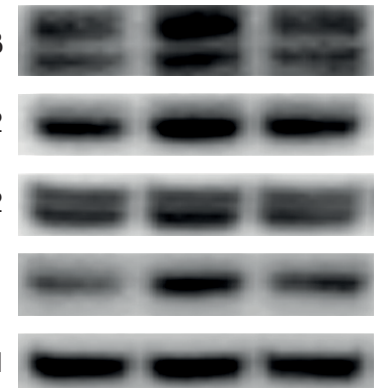

d)

Nuclei
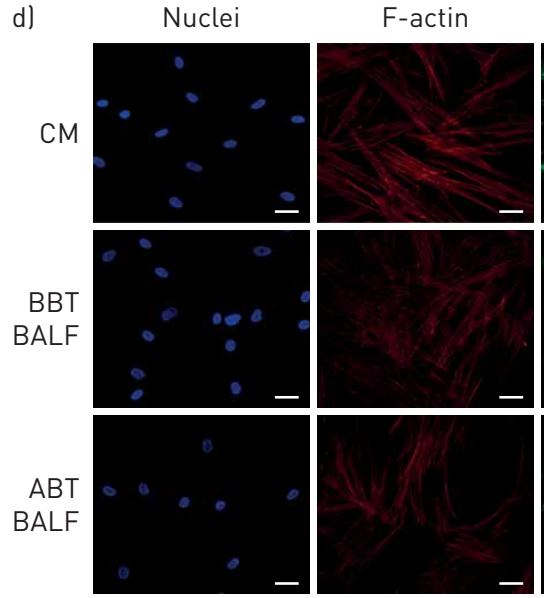
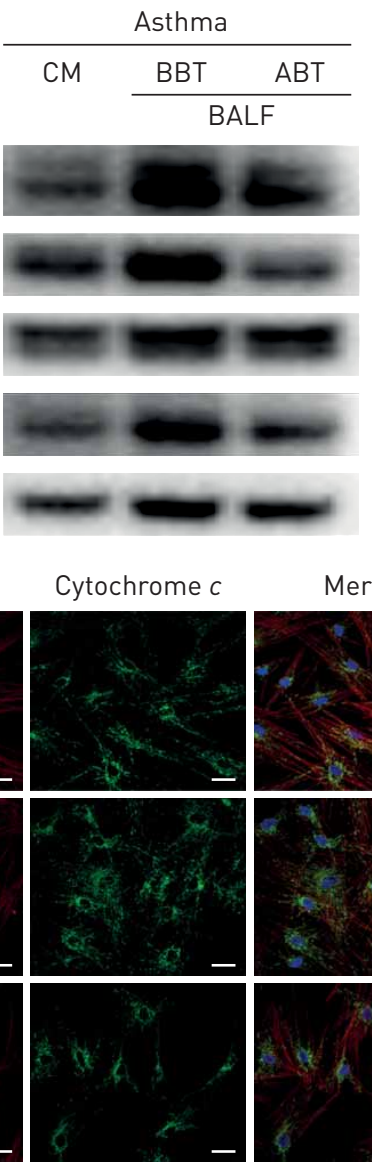
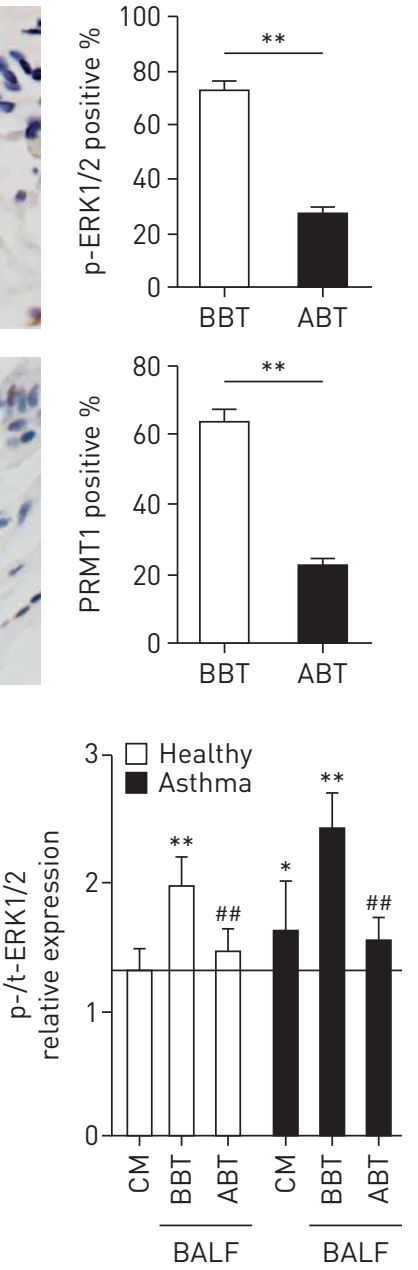
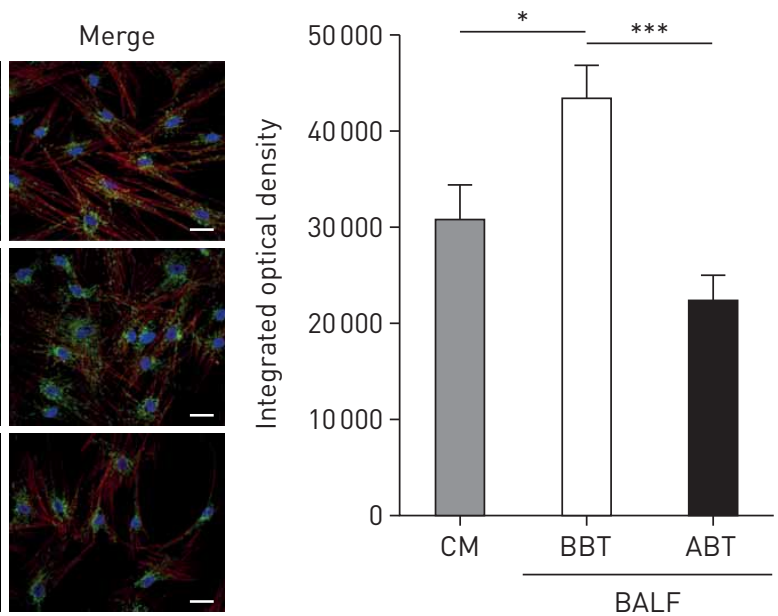

FIGURE 3 Bronchial thermoplasty (BT) reduced extracellular signal-regulated kinase-1/2 (ERK1/2) activation, protein arginine methyltransferase-1 (PRMT1) expression and mitochondrial mass in lung tissue and primary fibroblasts. BBT: before BT; ABT: after BT; miR-19a: microRNA-19a; CM: control medium; C/EBP $\beta$ : CCAAT enhancer-binding protein- $\beta$; $p$-: phosphorylated; $t$-: total; GAPDH: glyceraldehyde 3-phosphate dehydrogenase; BALF: bronchoalveolar lavage fluid. a) Representative immunohistochemistry of p-ERK1/2 mitogen-activated protein kinase (MAPK) and PRMT1 in airway tissue sections obtained BBT and ABT. Black arrows: epithelium; yellow arrows: mesenchymal cells. Bar charts summarise image analyses results. Data are presented as median and $95 \%$ confidence interval of the positive percentile ( $n=10$ ). Scale bars: $10 \mu \mathrm{m}$. *: $\mathrm{p}<0.05 ; * *: \mathrm{p}<0.01$. b) miR-19a expression in control fibroblasts stimulated with BALF from asthma patients obtained BBT or ABT. Data are presented as mean \pm SEM ( $n=9$ ). c) Representative Western blot of the stimulatory effect of BALF from asthma patients BBT and ABT on $p$-ERK1/2 MAPK, t-ERK1/2 MAPK and PRMT1 in fibroblasts. Bar charts summarise image analyses results. The horizontal lines indicate the level for CM. Data are presented as mean \pm SEM of five asthmatic and five nonasthma BALF samples, each tested in three fibroblast lines. ${ }^{*}: p<0.05 ;{ }^{* *}: p<0.01 ;{ }^{* * *}: p<0.001$, comparing healthy versus other fibroblasts; ${ }^{\#}: p<0.05 ;{ }^{\# \#}$ : $p<0.01$, comparing BBT versus ABT. d) Representative confocal microscopy images of the BALF effect on cytochrome $c$ and F-actin (phalloidin). Nuclei were stained by 4',6-diamidino-2-phenylindole (DAPI). Data are presented as mean \pm SEM ( $\mathrm{n}=11$ ) for cytochrome $c$ staining. Scale bars: $10 \mu \mathrm{m} .{ }^{*}: p<0.05 ; * * *: p<0.001$. 


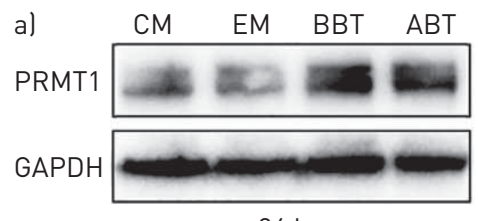

$24 \mathrm{~h}$

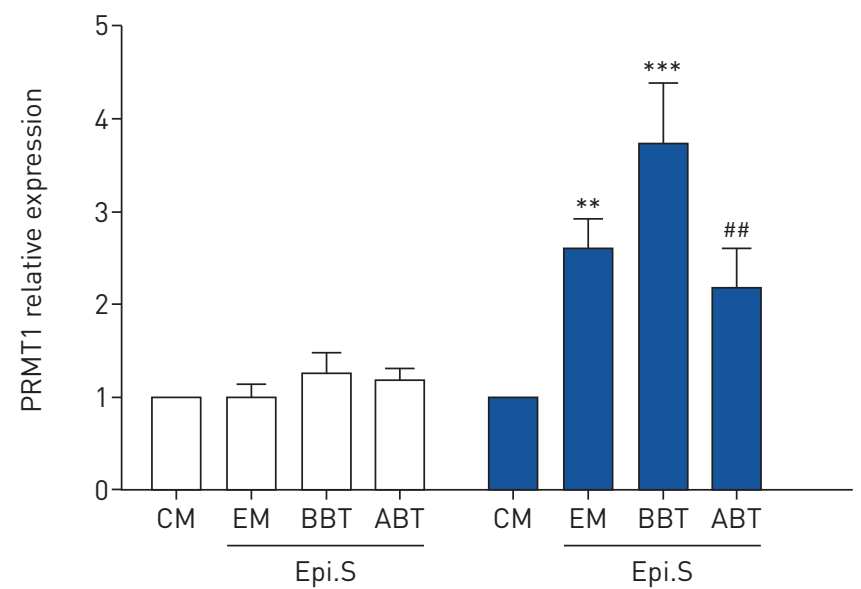

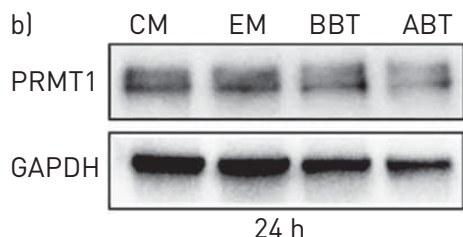
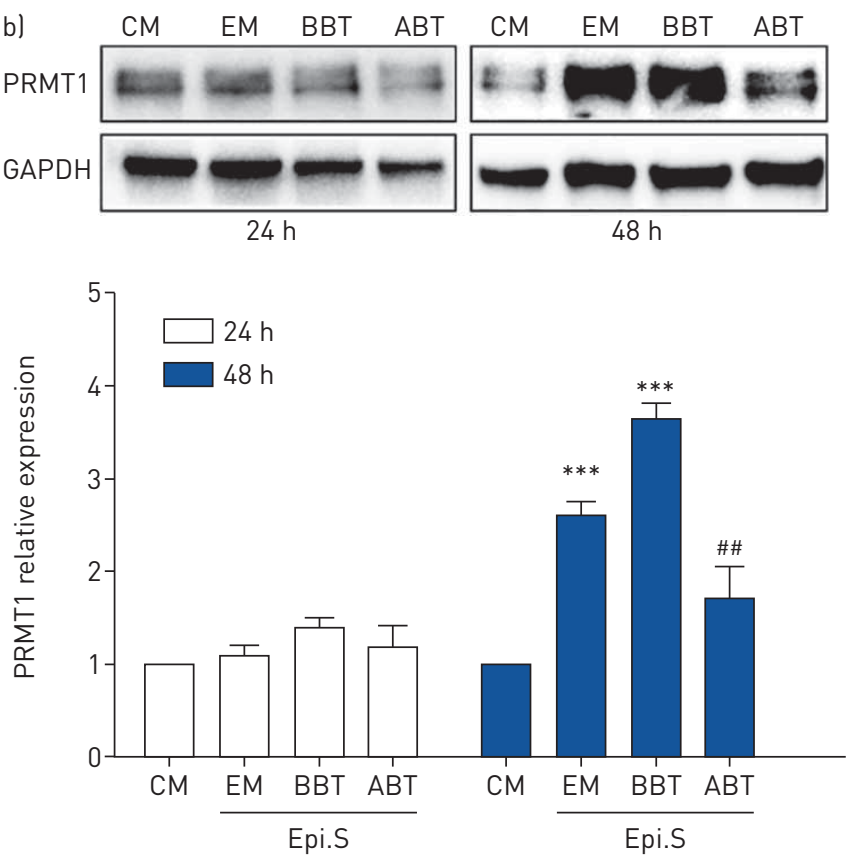

FIGURE 4 The effect of epithelial cell culture supernatant (Epi.S) obtained before and after bronchial thermoplasty (BBT and ABT, respectively) on protein arginine methyltransferase-1 (PRMT1) expression in fibroblasts: control fibroblast medium (CM), nonasthma epithelial cell culture medium (EM) or Epi.S obtained BBT and ABT was applied to primary fibroblasts from a) healthy or b) asthmatic donors. GAPDH: glyceraldehyde 3-phosphate dehydrogenase. Representative Western blots and quantitative image analysis of PRMT1 expression in fibroblasts of nonasthma and

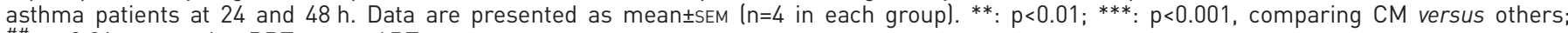
$\#$ : $p<0.01$, comparing BBT versus ABT.

Epi.S of cells obtained before BT significantly increased fibroblast proliferation (figure 6a) and their overall cell activity (figure 6b) when compared with Epi.S of cells obtained after BT. There was no difference comparing the response of fibroblasts from nonasthma with asthma cell donors and therefore the data were combined. The deposition of collagen type I and fibronectin was not different comparing Epi.S obtained before and after BT. Under both conditions, Epi.S significantly increased the deposition of collagen type I and fibronectin (figure $6 \mathrm{c}$ and $\mathrm{d}$ ).

\section{$B T$ reduced mitochondrial mass in fibroblasts by reducing $C / E B P \beta$ and PRMT1 expression}

Epi.S obtained before BT significantly increased the expression of the mitochondria activators C/EBP $\beta$, PGC1 $\alpha$ and PRMT1 by fibroblasts of healthy tissue donors (figure 7a; for quantitative analysis, see supplementary figure S5a). The stimulatory effect of Epi.S obtained before BT was significantly reduced in fibroblasts pre-treated with C/EBP $\beta$ siRNA, as shown by Western blot (figure 7a) and immunofluorescence (figure $7 \mathrm{~b}$ ). Downregulation of $\mathrm{C} / \mathrm{EBP} \beta$ also reduced the stimulatory effect of Epi.S obtained before BT on mitochondrial mass (MitoTracker) in control fibroblasts (figure 7b).

Furthermore, downregulation of C/EBP $\beta$ significantly reduced the proliferative effect of Epi.S obtained before BT on fibroblasts of asthma patients and controls (figure 7c).

Epi.S obtained before BT significantly upregulated C/EBP $\beta$, PRMT1, PGC1 $\alpha$, cytochrome $c$, HSP60 and $\alpha$-SMA expression by fibroblasts of both asthma patients and controls (figure $8 \mathrm{a}$; for quantitative analysis, see supplementary figure S5b). In fibroblasts treated with Epi.S obtained before BT, AMI-1 significantly reduced the expression of HSP60, cytochrome $c$ and $\alpha$-SMA (figure 8a), as well as C/EBP $\beta$ expression and MitoTracker signals (figure 8b). Similarly, downregulation of PRMT1 by siRNA reduced the expression of PGC1 $\alpha$ and C/EBP $\beta$ in control fibroblasts (figure 8c).

Blocking PRMT1 activity by AMI-1 also significantly reduced the pro-proliferative effect of Epi.S obtained before BT in fibroblasts from asthma patients and controls (figure 8d).

Proteomics analysis of BALF and NGS analysis in primary epithelial cells indicated that HSP6O expression is reduced after $B T$

14 BALF samples were analysed by high-resolution tandem mass spectrometry that included extensive peptide fractionation and phospho-peptide enrichment. In total, 1137 proteins were identified in the BALF (supplementary figures S3 and S4). Proteins with low abundance values (mean $<1$ ) were excluded before 
a)

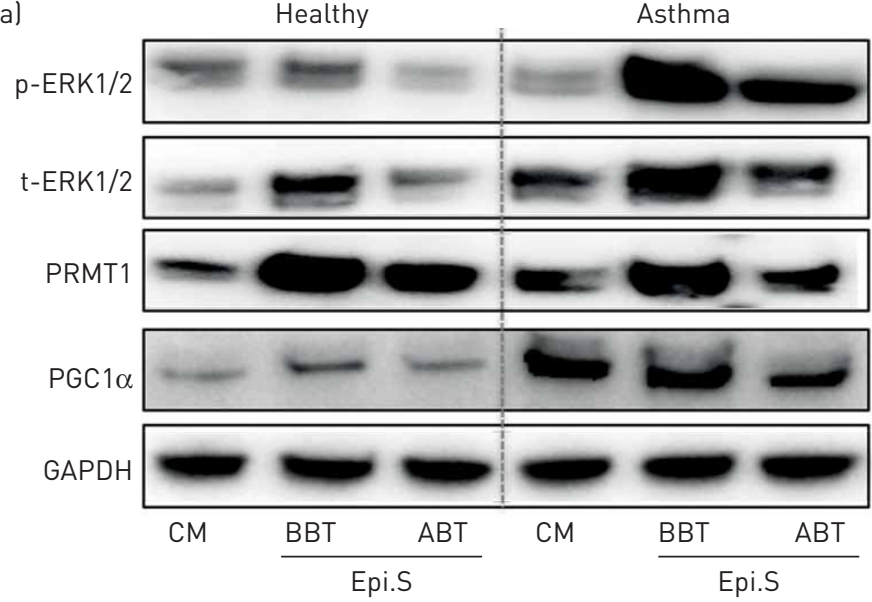

b)

$\mathrm{CM}$
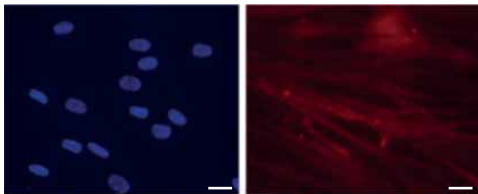

Epi.S
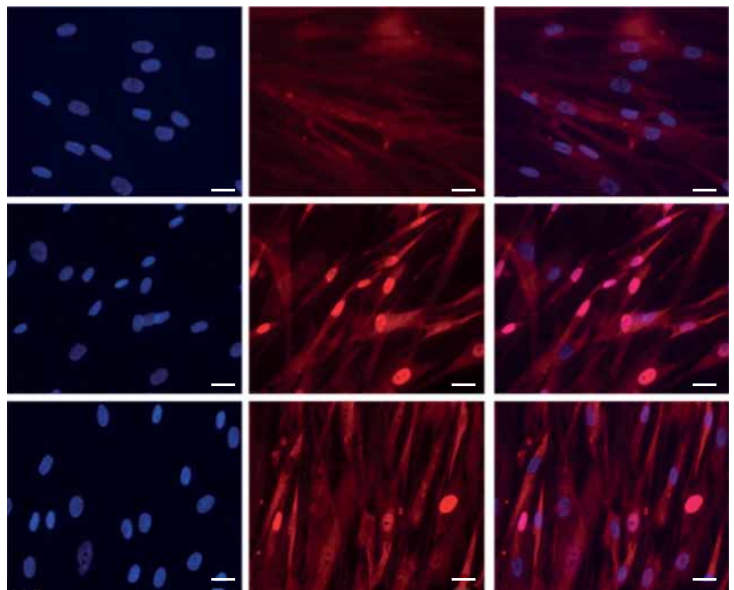

PRMT1/DAPI

Nuclei

d)

$\mathrm{CM}$

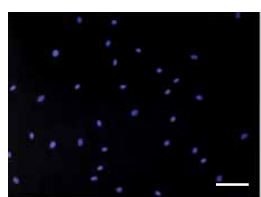

Epi.S
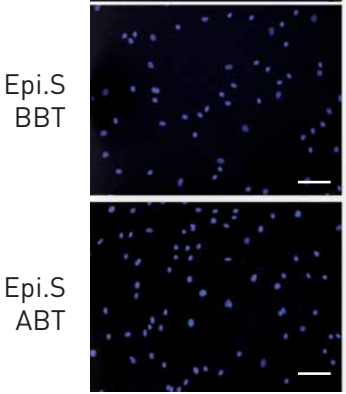

MitoTracker
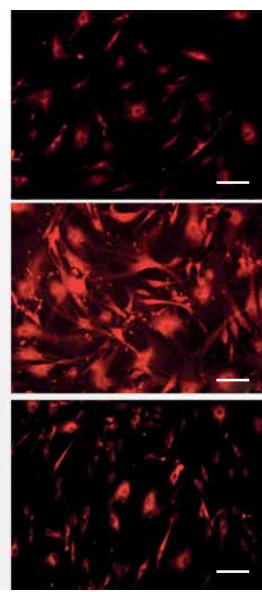

Merge
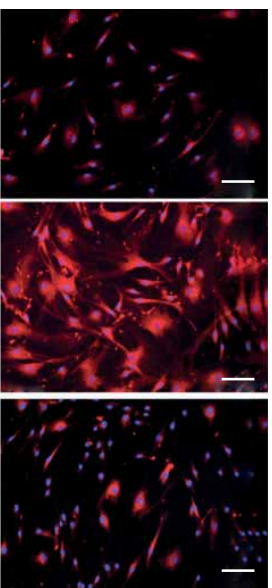

c)

$\mathrm{CM}$
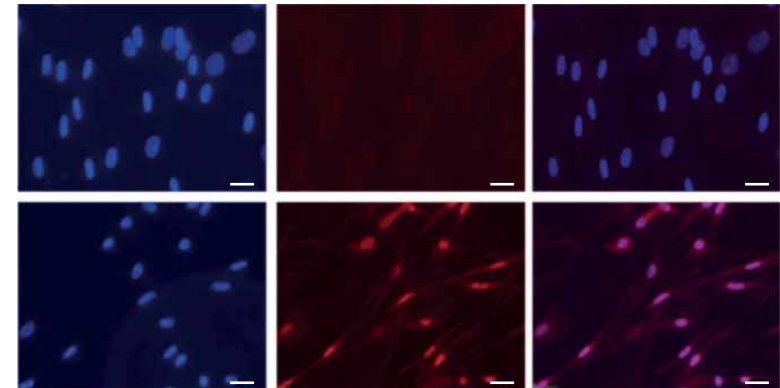

Epi.S

ABT
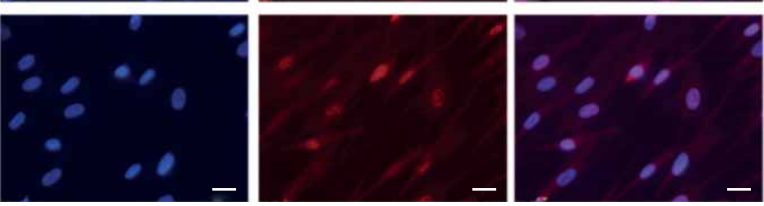

PGC1 $\alpha / D A P I$

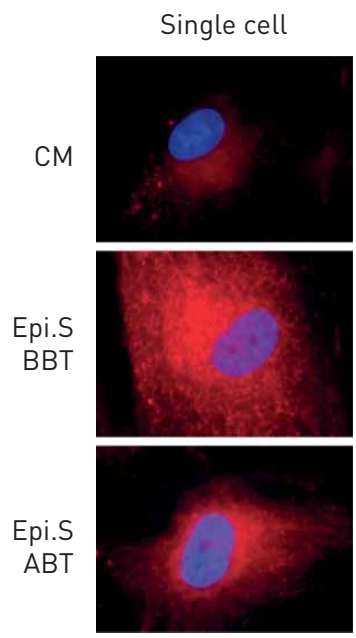

FIGURE 5 Bronchial thermoplasty (BT) reduced epithelial cell-induced increase of mitochondrial mass in fibroblasts. p-: phosphorylated; ERK1/2: extracellular signal-regulated kinase-1/2; t-: total; PRMT1: protein arginine methyltransferase-1; PGC1 $\alpha$ : peroxisome proliferator-activated receptor- $\gamma$ coactivator- $1 \alpha$; GAPDH: glyceraldehyde 3-phosphate dehydrogenase; CM: control medium; BBT: before BT; ABT: after BT; Epi.S: epithelial cell culture supernatant; DAPI: 4',6-diamidino-2-phenylindole. al ABT Epi.S did not induce the expression and phosphorylation of ERK1/2 mitogen-activated protein kinase ( $p$-ERK1/2 or t-ERK1/2), PRMT1 and PGC1 $\alpha$ compared with BBT Epi.S. Five Western blots of each group were analysed by image quantification. Data are presented as mean \pm SEM. ${ }^{*}: p<0.05 ;{ }^{* *}: p<0.01 ;{ }^{* * *}: p<0.001$. b, c) Representative immunofluorescence of b) nuclear PRMT1 accumulation and c) PGC1 $\alpha$ in healthy fibroblasts in the presence of BBT Epi.S and ABT Epi.S. d) Representative immunofluorescence of mitochondrial mass in healthy fibroblasts, determined by MitoTracker $48 \mathrm{~h}$ after exposure to BBT Epi.S and ABT Epi.S. Enlarged images of single cells are depicted in the right panel. Scale bars: $10 \mu \mathrm{m}$. 

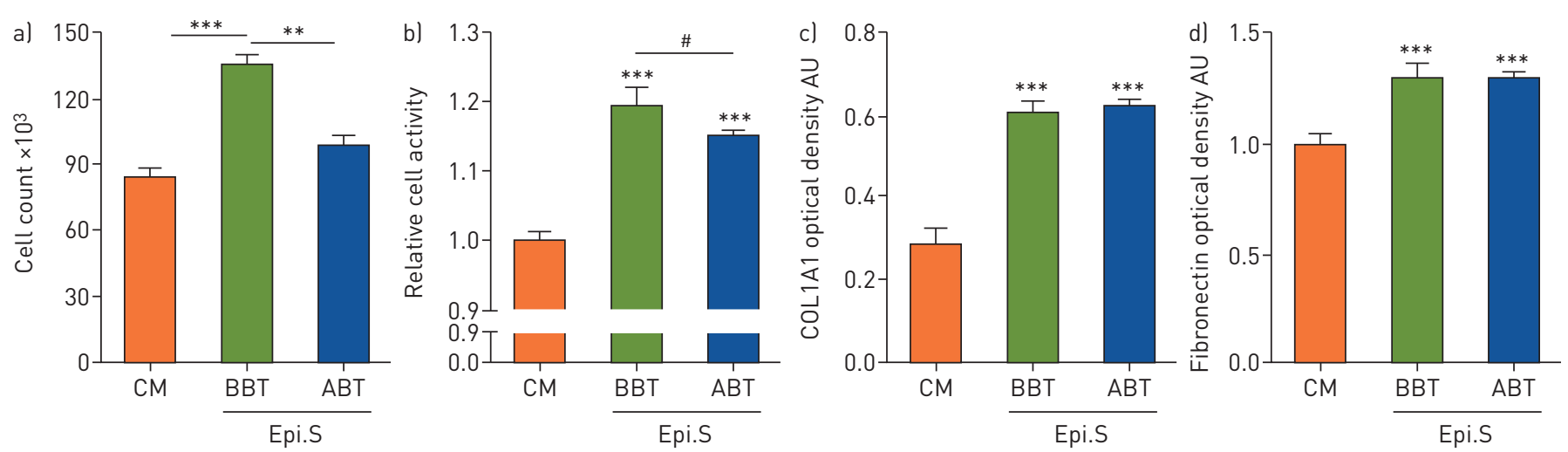

FIGURE 6 Bronchial thermoplasty (BT) reduced fibroblast proliferation and cell activation by bronchial epithelial cells. CM: control medium; BBT: before BT; ABT: after BT; Epi.S: epithelial cell culture supernatant; AU: arbitrary units. a) Direct cell counts after $48 \mathrm{~h}$ of incubating fibroblasts with either BBT Epi.S or ABT Epi.S $(n=6)$. b) Fibroblast activity measured by MTT (3-(4,5-dimethylthiazol-2-yl)-2,5-diphenyltetrazolium bromide) over $48 \mathrm{~h}(\mathrm{n}=4)$. c, d) BT had no effect on the deposition of $c$ collagen type I (COL1A1) ( $\mathrm{n}=4)$ or d) fibronectin ( $=4)$. Data are presented as mean \pm SEM. ${ }^{* *}: p<0.01 ;{ }^{* * *}$ : $p<0.001$, comparing control versus other groups; ${ }^{\#}: p<0.05$, comparing BBT versus ABT.
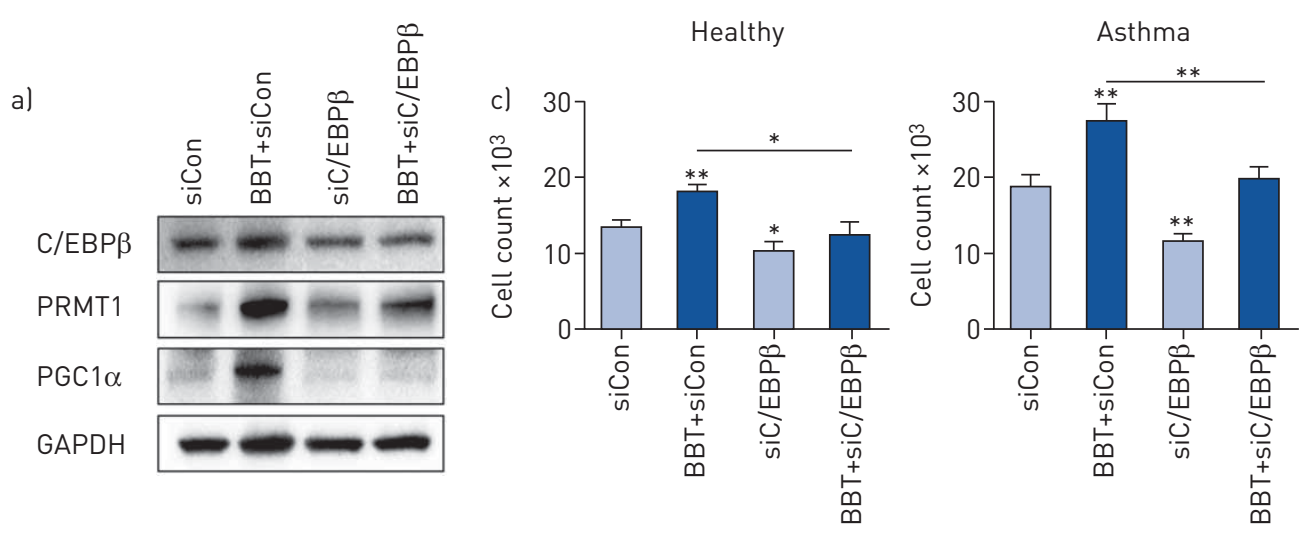

b)
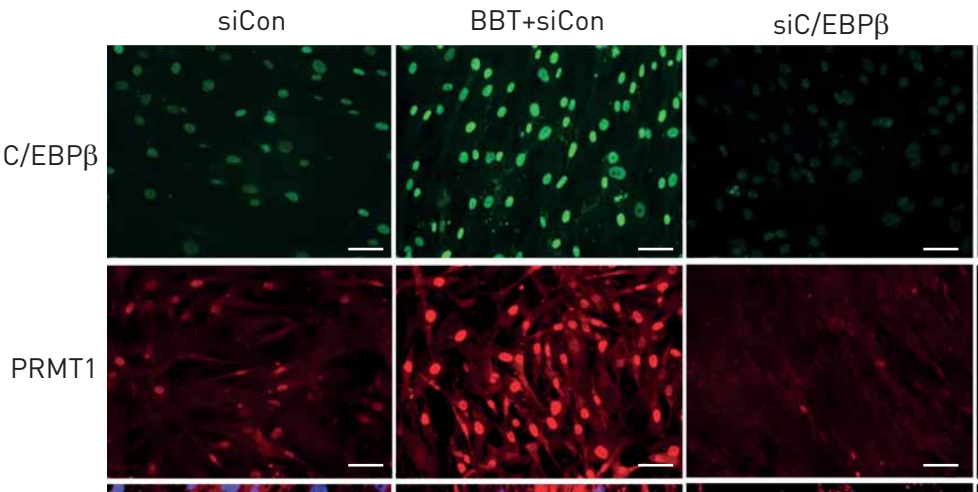

$\mathrm{BBT}+\mathrm{siC} / \mathrm{EBP} \beta$
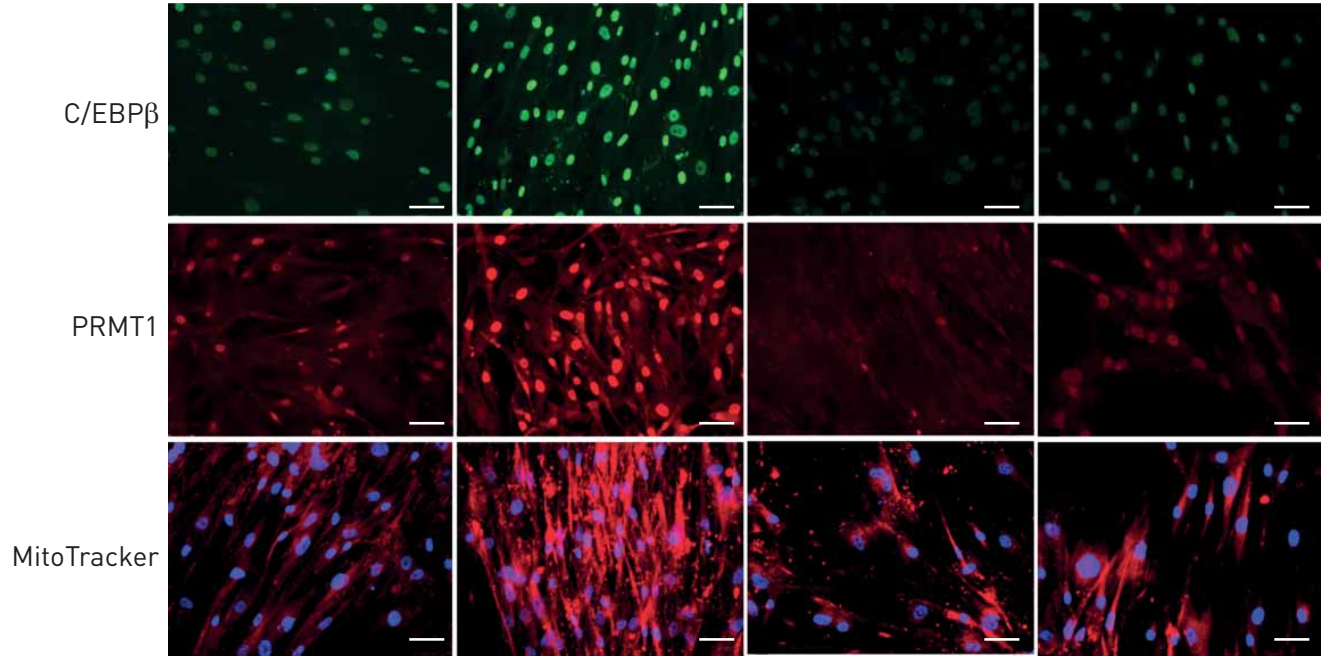

FIGURE 7 Bronchial thermoplasty (BT) reduced the stimulatory effect of epithelial cell culture supernatant (Epi.S) obtained before BT (BBT) on mitochondrial mass in fibroblasts through CCAAT enhancer-binding protein- $\beta$ (C/EBP $\beta$ ) and protein arginine methyltransferase-1 (PRMT1). PGC1 $\alpha$ : peroxisome proliferator-activated receptor- $\gamma$ coactivator-1 $\alpha$; GAPDH: glyceraldehyde 3-phosphate dehydrogenase; si: small interfering; Con: control. a) In healthy fibroblasts $(n=3)$ BBT Epi.S increased the expression of $C / E B P \beta, P G C 1 \alpha$ and PRMT1, which was downregulated by siRNA to C/EBP $\beta$ (siC/EBP $\beta$ ) within $48 \mathrm{~h}$. The quantitative analysis of all Western blots is provided in supplementary figure S5a. b) Representative immunofluorescence of healthy fibroblasts in the presence of siC/EBP $\beta$ with and without BBT Epi.S, showing the expression of C/EBP $\beta$, PRMT1 and MitoTracker. Scale bars: $10 \mu \mathrm{m}$. c) Downregulation of C/EBP $\beta$ significantly reduced the proliferative effect of BBT Epi.S on fibroblasts of asthma patients and controls $(n=3)$. Data are presented as mean \pm SEM. ${ }^{*}: p<0.05$; ${ }^{* *}$ : $p<0.01$, comparing control versus other groups. 


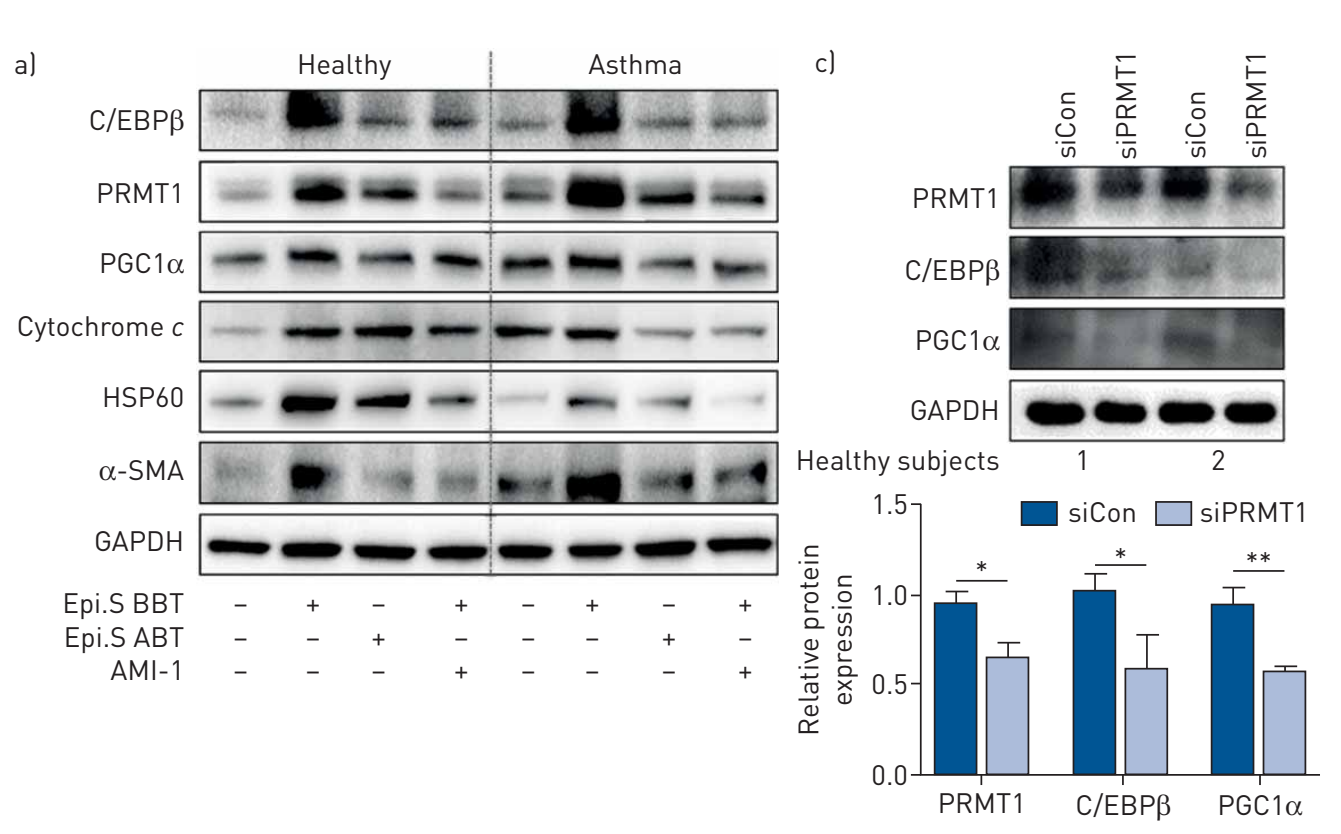

b)

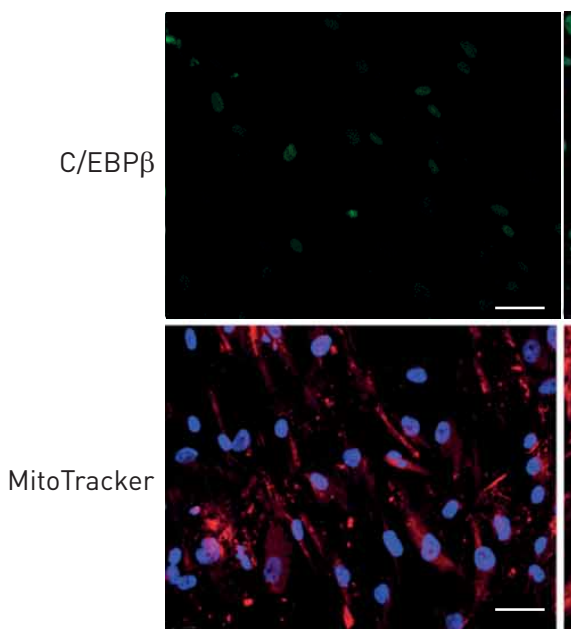

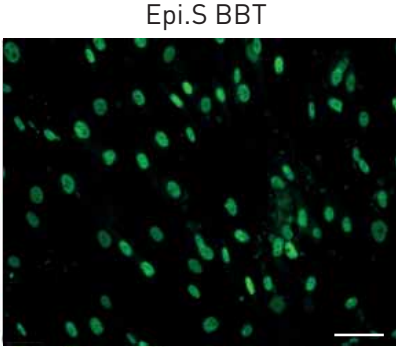

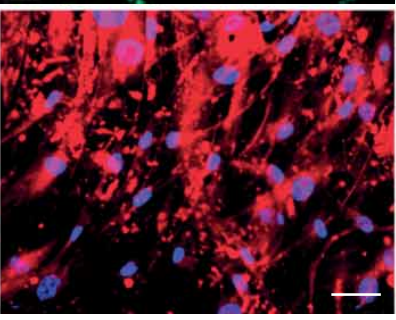

Epi.S ABT

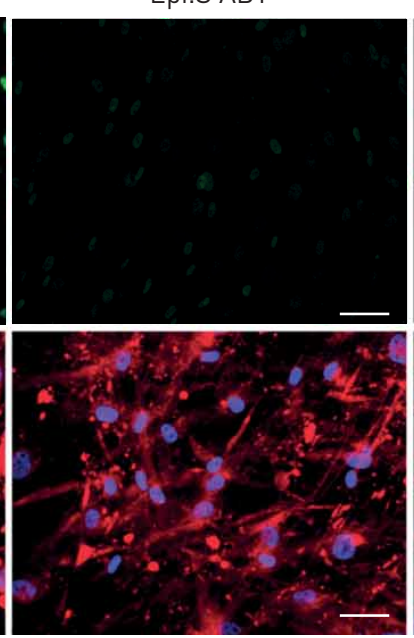

Healthy
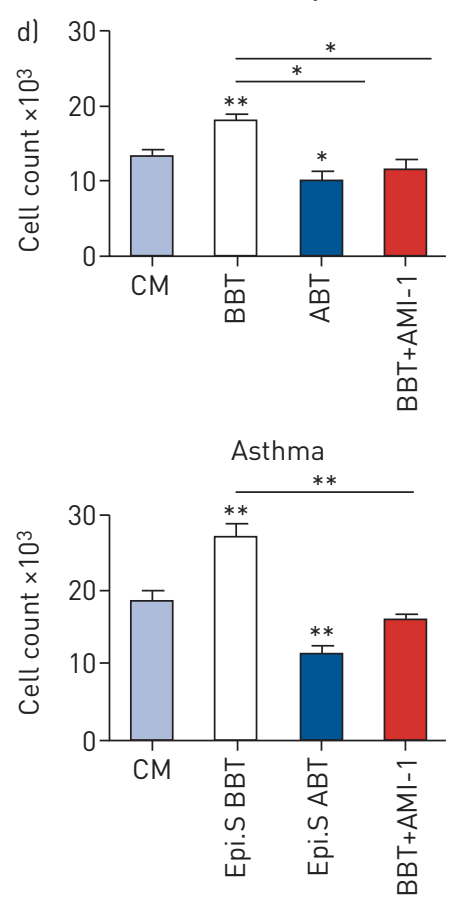

BBT+AMI-1
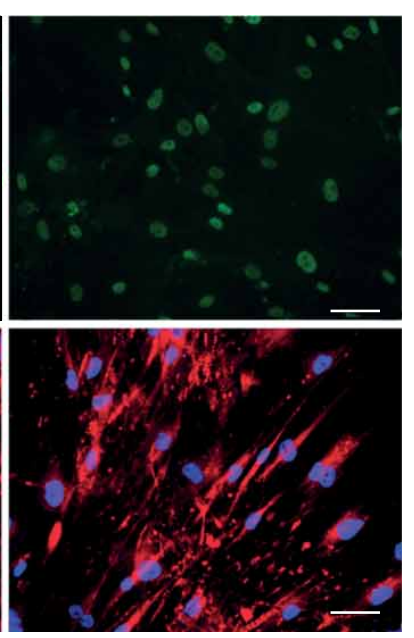

C/EBPß/MitoTracker/DAPI

FIGURE 8 Protein arginine methyltransferase-1 (PRMT1) inhibition reduced mitochondrial mass and regulators. C/EBP $\beta$ : CCAAT enhancer-binding protein- $\beta$; PGC1 $\alpha$ : peroxisome proliferator-activated receptor- $\gamma$ coactivator- $1 \alpha$; HSP60: heat shock protein-60; $\alpha$-SMA: smooth muscle actin; GAPDH: glyceraldehyde 3-phosphate dehydrogenase; Epi.S: epithelial cell culture supernatant; BT: bronchial thermoplasty; BBT: before BT; ABT: after BT; si: small interfering; Con: control; CM: control medium. al BBT Epi.S-induced expression of mitochondria activators was reduced by AMI-1. The quantitative analysis of all Western blots ( $n=4$ in each group) is provided in supplementary figure S5b. b) Representative immunofluorescence of BBT Epi.S-induced expression of C/EBP $\beta$ and MitoTracker in the presence and absence of AMI-1. Scale bars: $10 \mu m$. c) Representative Western blot of siRNA for PRMT1 (siPRMT1) on the expression of PGC1 $\alpha$ and C/EBP $\beta$. The quantitative analysis of all Western blots ( $n=3)$ is shown in the bar chart below the blot. d) The effect of AMI-1 on BBT Epi.S-induced fibroblast proliferation ( $n=3)$. Data are presented as mean \pm SEM. *: $\mathrm{p}<0.05 ;{ }^{* *}$ : $\mathrm{p}<0.01$, comparing control versus other groups.

data analysis by OmicShare tools. Compared with BALF obtained before BT, 49 differentially expressed proteins (DEPs) were identified in BALF obtained after BT. Among these, 28 DEPs were upregulated and 21 DEPs were downregulated (table 2). Most significantly, HSP60 (HSPD1) was downregulated in BALF obtained after BT compared with BALF obtained before BT; in contrast, mucin (MUC5A) and complement C5 were upregulated (figure 9a).

To determine the effect of BT on protein/gene regulatory pathways, KEGG analysis was performed. KEGG analysis suggested that most BT-regulated DEPs were linked to signal transduction (EEF2, YWHAE, FN1, 
TABLE 2 Mass spectrometric analysis of the identified differentially expressed proteins in bronchoalveolar lavage fluid from patients before and after bronchial thermoplasty (BBT and ABT)

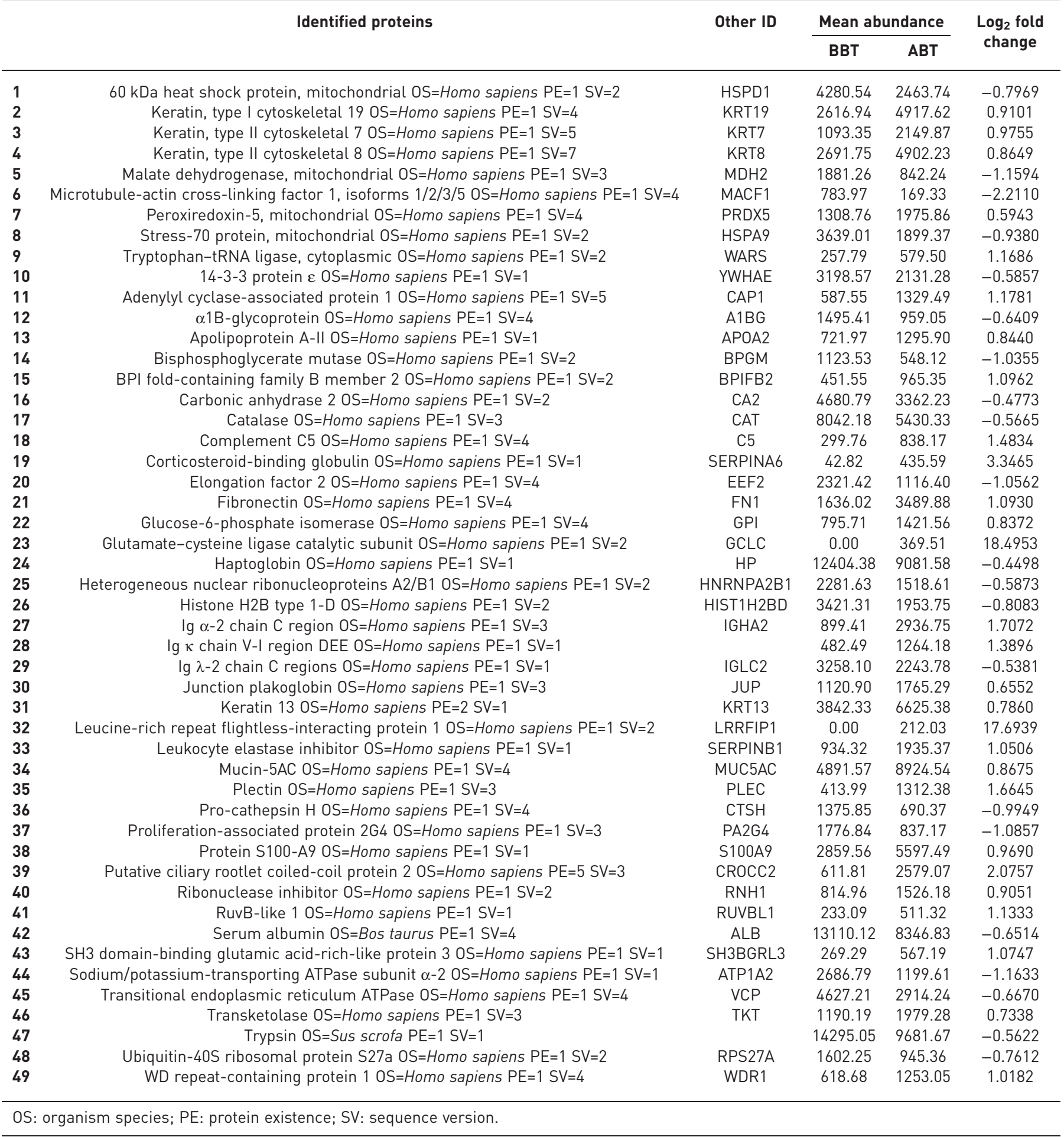

CAT, RUVBL1 and ATP1A2), infectious diseases (HSPD1, VCP, SERPINB1, FN1, HSPA9, C5 and YWHAE) and the immune system (S100A9, MUC5AC and C5) (figure $9 \mathrm{~b}$ and c, and table 3).

Transcriptome analysis of primary epithelial cells confirmed the modification of HSP60 expression (table 4). Therefore, the role of HSP60 in the regulation of PRMT1 expression was further assessed in lung fibroblasts. Stimulation of fibroblasts with human recombinant HSP60 $\left(1 \mu \mathrm{g} \cdot \mathrm{mL}^{-1}\right)$ significantly increased 
a)

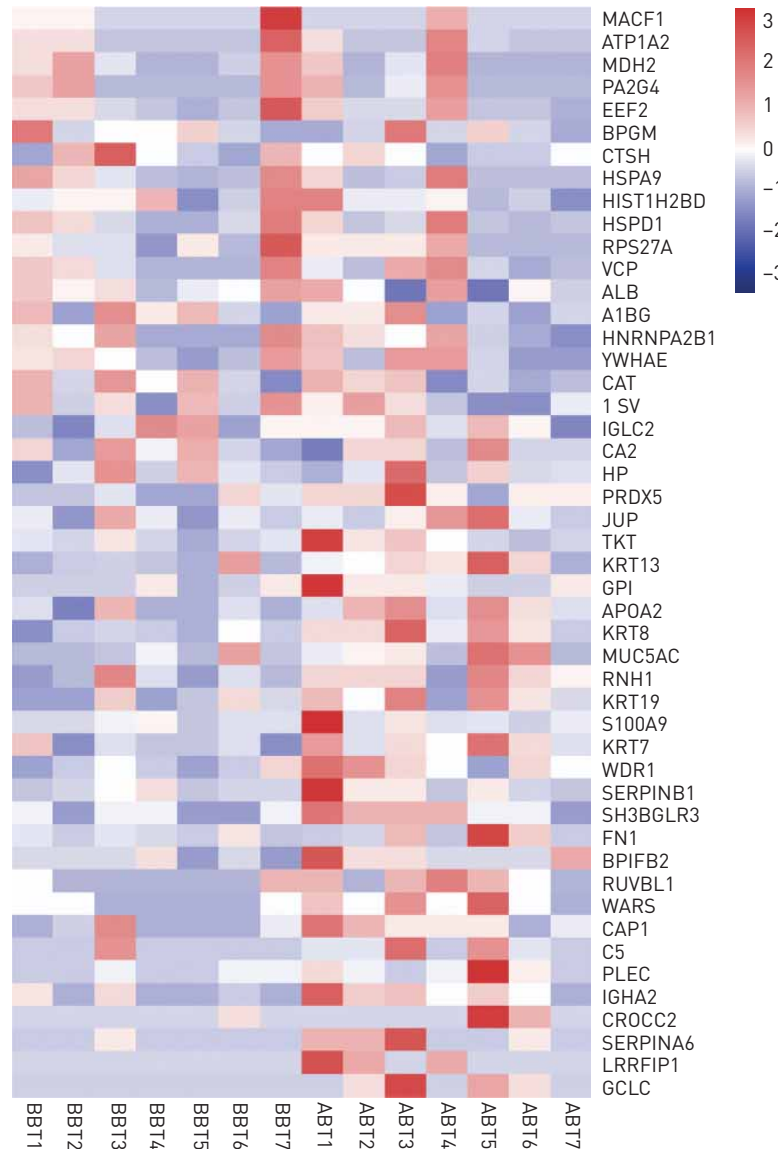

c)

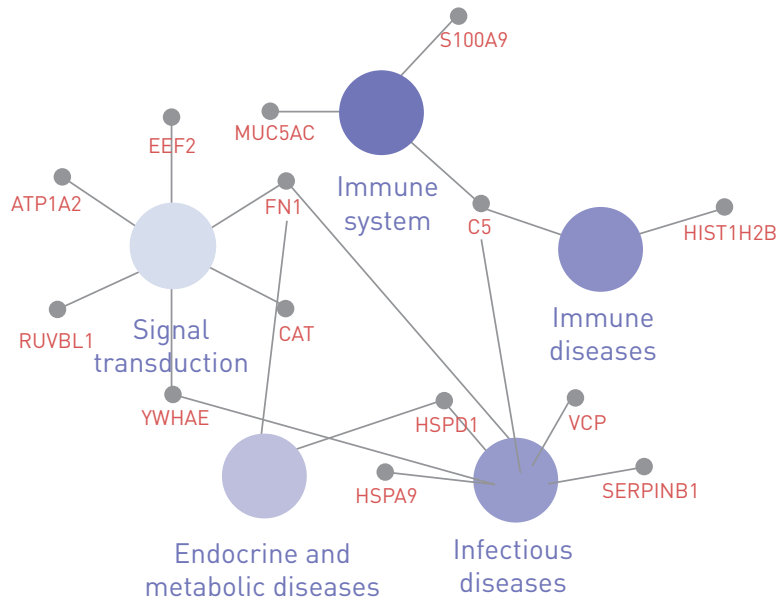

d)

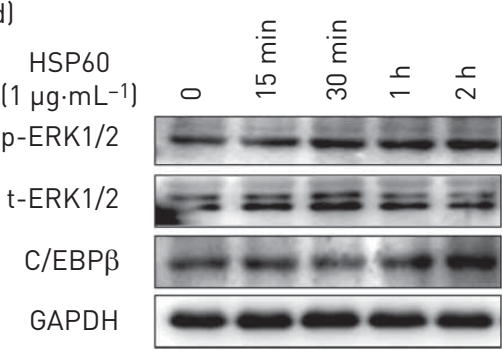

e)

HSP60

$\left(1 \mu \mathrm{g} \cdot \mathrm{mL}^{-1}\right) \quad 0 \quad 48 \mathrm{~h}$

C/EBP $\beta=$

PRMT1

PGC1 $\rightleftharpoons \ldots$

GAPDH

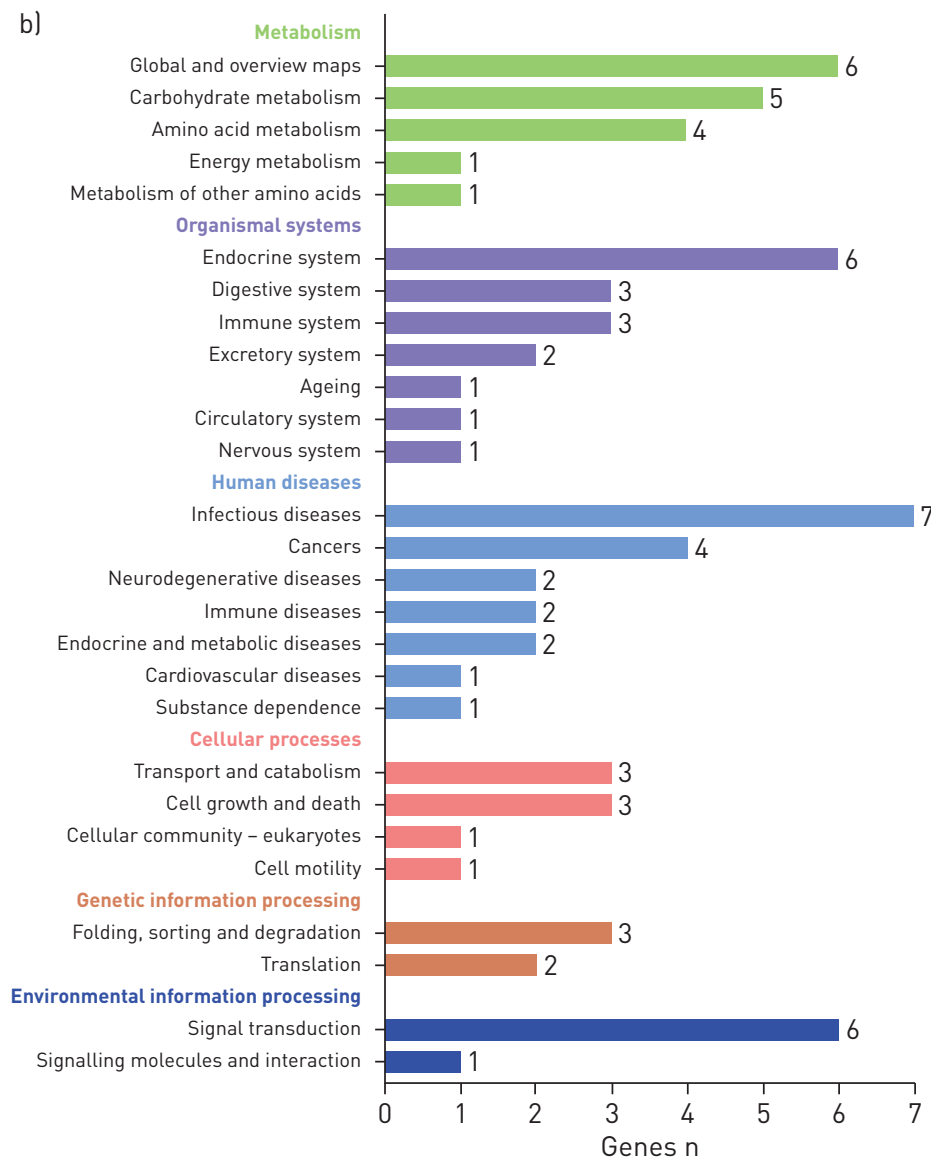

f)

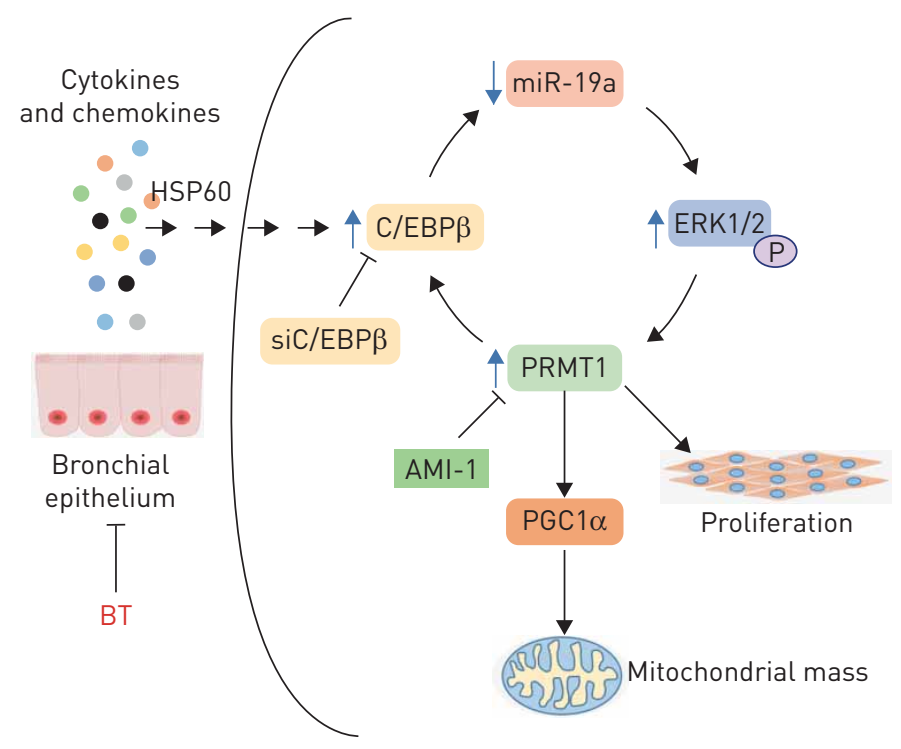


FIGURE 9 Proteomics analysis of bronchoalveolar lavage fluid (BALF) using the OmicShare tools indicated that heat shock protein-60 (HSP60) controls protein arginine methyltransferase-1 (PRMT1) expression. BT: bronchial thermoplasty; BBT: before BT; ABT: after BT; $p$-: phosphorylated; ERK1/2: extracellular signal-regulated kinase-1/2; $t$-: total; C/EBP $\beta$ : CCAAT enhancer-binding protein- $\beta$; GAPDH: glyceraldehyde 3-phosphate dehydrogenase; PGC1 $\alpha$ : peroxisome proliferator-activated receptor- $\gamma$ coactivator-1 $\alpha$; miR-19a: microRNA-19a; si: small interfering. a) Heat map of differentially expressed proteins contained in BBT BALF from seven patients compared with ABT BALF. Each row shows the expression of a single protein and each column represents a single BALF sample. The colour scale indicates high, medium and low protein expression. b) Kyoto Encyclopedia of Genes and Genomes analysis of 49 differentially expressed proteins (table 3). c) Cytoscape map of representative signalling pathway networks and related genes. d) Kinetics of HSP60-stimulated p-ERK1/2, t-ERK1/2 and C/EBP $\beta$ expression in control fibroblasts demonstrated by Western blot. The quantitative analysis of all Western blots is provided in supplementary figure S5c. el Primary fibroblasts of control patients $(\mathrm{n}=3)$ were treated with $1 \mu \mathrm{g} \cdot \mathrm{mL}^{-1}$ recombinant human HSP60 for $48 \mathrm{~h}$. The expression of C/EBP $\beta$, PRMT1 and PGC $1 \alpha$ was detected by Western blot. The quantitative analysis of all Western blots is provided in supplementary figure S5c. f) Graphic summary of the study showing that BT interrupts pro-remodelling signalling through PRMT1.

the C/EBP $\beta$ and ERK1/2 MAPK signalling cascade within $2 \mathrm{~h}$, which stimulated the expression of PRMT1 and PGC1 $\alpha$ at $48 \mathrm{~h}$ (figure 9d and e; for quantitative Western blot analysis, see supplementary figure S5c).

Figure $9 \mathrm{f}$ summarises the interaction of epithelial cells and fibroblasts, and its modification by BT.

\section{Discussion}

BT is the only therapy shown to reduce remodelling in patients with severe asthma; however, the mechanism is unknown $[6,7,12]$. This study provides the first evidence that BT produces lasting effects on bronchial epithelial cell function and thereby changes some remodelling properties of airway wall fibroblasts. BALF and Epi.S of cells isolated from patients with severe asthma before BT reduced the expression of miR-19a in fibroblasts, thereby stimulating the expression of ERK1/2 MAPK, PGC1 $\alpha$ and PRMT1, and thus increasing mitochondrial mass and fibroblast proliferation. Importantly, these effects were lost after BT.

In an animal asthma model, PRMT1 was upregulated first in epithelial cells and later in mesenchymal cells, by cell type specific cytokines $[19,20,22]$. In tissue sections of asthma patients obtained before BT, phosphorylation of ERK1/2 MAPK and PRMT1 expression were high in epithelial cells and lower in

TABLE 3 Kyoto Encyclopedia of Genes and Genomes (KEGG) pathways of differentially expressed proteins from bronchoalveolar lavage fluid

\section{KEGG A class}

Metabolism

Organismal systems

Human diseases

Cellular processes

Genetic information processing

Environmental information processing

\section{KEGG B class}

Global and overview maps

Carbohydrate metabolism

Amino acid metabolism

Energy metabolism

Metabolism of other amino acids

Endocrine system

Digestive system

Immune system

Excretory system Ageing

Circulatory system

Nervous system

Infectious diseases

Cancers

Neurodegenerative diseases Immune diseases

Endocrine and metabolic diseases

Cardiovascular diseases

Substance dependence

Transport and catabolism

Cell growth and death

Cellular community - eukaryotes Cell motility

Folding, sorting and degradation Translation

Signal transduction

Signalling molecules and interaction
Proteins $\mathbf{n}$

Proteins/genes 
TABLE 4 Transcriptome analysis

\begin{tabular}{|c|c|c|c|c|}
\hline Gene & $\log _{2}$ fold change & Log CPM & p-value & False discovery rate \\
\hline HSPA7 & -6.6745634 & 2.94357766 & $2.8625 \mathrm{E}-56$ & $4.325 E-52$ \\
\hline CCL26 & -4.0022854 & 1.53369684 & $4.5982 E-36$ & 3.4737E-32 \\
\hline RN7SKP48 & -8.4063178 & 0.00054165 & $4.2481 \mathrm{E}-34$ & $2.1395 \mathrm{E}-30$ \\
\hline$A R C$ & -6.0359691 & 2.80438403 & $1.3802 E-29$ & $5.2134 \mathrm{E}-26$ \\
\hline ANKRD1 & -3.5612232 & 2.00265271 & $1.2001 E-17$ & $3.6264 E-14$ \\
\hline CRYAB & -1.4560917 & 7.69968071 & $1.2704 \mathrm{E}-15$ & $3.199 \mathrm{E}-12$ \\
\hline HSPA6 & -6.1119842 & 5.60205649 & $1.0489 E-14$ & $2.264 \mathrm{E}-11$ \\
\hline VTRNA1-3 & -5.0654191 & 2.21568985 & 8.9014E-13 & $1.6811 \mathrm{E}-09$ \\
\hline SPP1 & -2.8175604 & 0.80469766 & $3.4552 \mathrm{E}-12$ & 5.8005E-09 \\
\hline HSPA1B & -3.291132 & 8.25091346 & $1.2859 E-10$ & $1.9428 \mathrm{E}-07$ \\
\hline XIRP1 & -4.3644087 & 0.39829287 & $4.8336 \mathrm{E}-10$ & $6.6391 \mathrm{E}-07$ \\
\hline NEFM & -2.8494359 & 2.86373374 & $9.6932 E-10$ & $1.2205 E-06$ \\
\hline FOSB & -2.3239735 & 2.72522439 & 2.473E-09 & $2.8742 E-06$ \\
\hline RRAD & -2.2480685 & 4.46413378 & $5.0727 \mathrm{E}-09$ & $5.4745 \mathrm{E}-06$ \\
\hline HSPA1A & -2.5310616 & 8.45620337 & $2.8287 E-08$ & $2.8492 \mathrm{E}-05$ \\
\hline ILIRL1 & -2.2992064 & 1.98026678 & 6.8739E-08 & $6.4911 \mathrm{E}-05$ \\
\hline RASD1 & -2.2417413 & 1.63816909 & $8.5819 \mathrm{E}-08$ & 7.6273E-05 \\
\hline VTRNA1-2 & -4.2152004 & 2.37699757 & $2.3762 \mathrm{E}-07$ & 0.00019946 \\
\hline IL16 & 0.9270627 & 3.3688119 & $3.1214 \mathrm{E}-07$ & 0.00024822 \\
\hline SPON1 & 0.76087863 & 5.63037004 & $1.4456 \mathrm{E}-06$ & 0.00109207 \\
\hline COL15A1 & 1.0153401 & 6.86806305 & $1.5531 \mathrm{E}-06$ & 0.00111743 \\
\hline$R P 11-96 K 19.4$ & -2.3607353 & -0.5695676 & $1.8955 \mathrm{E}-06$ & 0.0012725 \\
\hline$A B C A 8$ & 0.6821761 & 4.63139662 & $1.9371 \mathrm{E}-06$ & 0.0012725 \\
\hline HIST1H2BK & -0.7249494 & 4.90831468 & $2.3208 E-06$ & 0.00146106 \\
\hline HBEGF & -1.0262606 & 2.6512004 & $2.5521 \mathrm{E}-06$ & 0.00150211 \\
\hline UPP1 & -0.6218694 & 4.65882924 & $2.5849 \mathrm{E}-06$ & 0.00150211 \\
\hline MASP1 & 0.81818236 & 7.05487073 & $9.1183 \mathrm{E}-06$ & 0.00510251 \\
\hline$C 1 R L$ & 0.44446462 & 5.41931433 & $1.1084 \mathrm{E}-05$ & 0.00598114 \\
\hline DUSP5 & -0.4901746 & 4.66863971 & 1.2827E-05 & 0.00668276 \\
\hline$T N X B$ & 0.57515764 & 5.54831679 & $1.3871 \mathrm{E}-05$ & 0.00698593 \\
\hline THBD & -0.5948758 & 5.0121393 & $2.0903 E-05$ & 0.01018796 \\
\hline ADAMTS8 & 0.79724554 & 5.51175195 & $2.3182 \mathrm{E}-05$ & 0.01065668 \\
\hline MXRA5 & 0.62849027 & 7.08606778 & $2.3276 \mathrm{E}-05$ & 0.01065668 \\
\hline DUSP1 & -0.7788436 & 7.04579431 & $2.7687 \mathrm{E}-05$ & 0.01230367 \\
\hline MMP12 & -2.2223219 & 3.62749486 & $2.9065 \mathrm{E}-05$ & 0.01254702 \\
\hline SLITRK6 & 0.892711 & 5.72816647 & 3.1127E-05 & 0.01306376 \\
\hline LINC00324 & -2.2447718 & 0.41686191 & 3.659E-05 & 0.01494156 \\
\hline PLXDC2 & 0.52042951 & 5.75171529 & 4.2403E-05 & 0.01685985 \\
\hline$A B C A 6$ & 0.7034962 & 3.44538556 & 4.5537E-05 & 0.01764146 \\
\hline$R P 11-64 B 16.2$ & -2.7001276 & -0.3550856 & $4.9958 \mathrm{E}-05$ & 0.01887036 \\
\hline LAMA4 & 0.62652521 & 8.74391096 & $5.2261 \mathrm{E}-05$ & 0.01925895 \\
\hline VTRNA2-1 & -5.2769676 & 0.22835068 & $5.4686 \mathrm{E}-05$ & 0.01928778 \\
\hline METTL7A & 0.47147354 & 5.2757973 & $5.4893 \mathrm{E}-05$ & 0.01928778 \\
\hline LCE3D & -1.2948825 & 1.28528292 & $5.8792 \mathrm{E}-05$ & 0.02018826 \\
\hline WISP1 & 0.823069 & 3.56387389 & $6.5606 \mathrm{E}-05$ & 0.02183693 \\
\hline LCE3E & -1.2785048 & 1.1649956 & 6.6483E-05 & 0.02183693 \\
\hline GADD45B & -1.2909076 & 5.87248869 & $7.0692 \mathrm{E}-05$ & 0.02272509 \\
\hline RGS16 & -1.3795921 & 0.16021976 & 8.2909E-05 & 0.02609742 \\
\hline$R P 11-367 F 23.2$ & -2.5075731 & 0.32741435 & $8.6844 \mathrm{E}-05$ & 0.026778 \\
\hline AF131217.1 & 0.51616864 & 4.24716742 & $9.5197 \mathrm{E}-05$ & 0.02876658 \\
\hline
\end{tabular}

CPM: counts per million. Nine primary epithelial cell isolates obtained before and after experimental bronchial thermoplasty were exposed to $37^{\circ} \mathrm{C}$ versus $65^{\circ} \mathrm{C}$ and next-generation sequencing was performed as described in the Methods section. Transcripts with the highest fold change between groups are shown at the top of the table. p-values have been adjusted using the Benjamini-Hochberg false discovery rate to correct for multiple testing.

subepithelial mesenchymal cells. ERK1/2 MAPK phosphorylation and PRMT1 expression were lost in tissue sections obtained after BT. It should be noted that the tissue biopsies were obtained 4-12 weeks after BT and thus the effect was lasting. Our findings suggest a central role for PRMT1 in the regulation of asthma-associated airway fibroblast remodelling. 
Bronchial epithelial cells isolated after BT proliferated significantly faster than epithelial cells isolated from the same patients before BT, indicating improved epithelium repair capacity. These findings may explain improved re-epithelisation and structure adjustment, which were reported in clinical studies after BT [12, 24]. The response of fibroblasts to BALF or Epi.S suggested that the beneficial effect of BT depends on the modified function of epithelial cells.

In human asthmatic mesenchymal cells, the constitutive expression of PRMT1 was caused by decreased levels of miR-19a and upregulated ERK1/2 MAPK, subsequently activating signal transducer and activator of transcription-1 (STAT1) that increased both PRMT1 expression and mitochondrial mass [17, 21, 23]. Similarly, BALF and Epi.S obtained before BT downregulated miR-19a, leading to increased ERK1/2 MAPK in fibroblasts. This study also confirmed that C/EBP $\beta$ is a negative regulator of miR-19a, as reported in other cell types $[24,25]$. Furthermore, our observations suggest a feedback mechanism $(\mathrm{C} / \mathrm{EBP} \beta \rightarrow$ miR-19a $\rightarrow \mathrm{ERK} 1 / 2 \quad \mathrm{MAPK} \rightarrow \mathrm{PRMT} 1 \rightarrow \mathrm{C} / \mathrm{EBP} \beta)$ leading to fibroblast remodelling in asthma. Most importantly, such a mechanism can be lastingly broken by BT.

Mitochondria function and biogenesis were required to drive airway wall remodelling in asthma and COPD [26-28]. Here, the mitochondria regulating proteins PGC1 $\alpha$, cytochrome $c$ and HSP60 were increased in fibroblasts by Epi.S obtained before BT and were downregulated after BT.

In vitro, BT reduced smooth muscle mass and $\alpha$-SMA in myofibroblasts [13]. SALEM et al. [5] described that BT, over a period of 27 months, reduced airway smooth muscle cell mass, $\alpha$-SMA expression and basement membrane thickness in 10 patients with severe asthma. In this study, Epi.S obtained before BT increased $\alpha$-SMA expression and mitochondrial mass in fibroblasts, and these effects were lost in Epi.S obtained after BT. This observation may help to explain the lasting reduction of subepithelial mesenchymal cell numbers reported after BT $[5,11,12]$. The fact that in this study epithelial cells isolated after BT lost their stimulatory properties suggests that BT induced a lasting "re-programming".

Proteome analysis of BALF and transcriptome analyses in isolated epithelial cells suggested that HSP60 mediated most BT effects. HSP60 contributed to inflammation by activating immune cells [29] and inducing cell dysfunction in obese patients $[30,31]$. In this study, exogenous HSP60 increased PRMT1 expression, the corresponding signalling cascade and mitochondrial mass in fibroblasts. Our results indicated that BALF and Epi.S of asthma patients obtained before BT caused mitochondrial dysfunction through upregulation of PGC1 $\alpha \rightarrow$ PRMT1, which is consistent with earlier reports $[32,33]$.

The strength of this study is to provide a molecular biological mechanism that explains the lasting beneficial effect of BT on some parameters of airway wall remodelling.

The limitations of this study include: 1) further studies assessing the consequences of BT-induced changes on protein expression on target cell function, such as smooth muscle cells, have to be performed; 2) the observed BT-induced changes in the proteome and transcriptome should be confirmed in tissue sections from a larger number of asthma patients; 3 ) mass spectrometry could not be performed for all conditions, due to the presence of bovine albumin in the cell culture medium which obscured the reading (therefore we were not able to confirm that the secreted factors found in BALF originated from epithelial cells); and 4) we could not investigate all BT-induced changes due to limited availability of tissue sections after BT.

In conclusion, we present a novel self-sustaining, remodelling driving mechanism in fibroblasts of asthma patients. Importantly, this mechanism could be lastingly broken by BT. Furthermore, the findings suggest that HSP60, miR-19a or PRMT1 may present new therapeutic targets to control airway wall remodelling in asthma.

Acknowledgements: We are grateful to Xinggang Wang (Reproductive Medical Centre, Jiaotong University, Shanghai, China) and Hui Wang (Stem Cell Research, University Hospital Basel, Basel, Switzerland) for their expertise assistance, helpful discussions and productive critiques. We also thank C.T. S’ng (PersCellMed S'ng, Sissach, Switzerland) for his help in preparing this manuscript.

Author contributions: Q. Sun, L. Fang, D. Stolz, S. Lu and M. Roth conceived and designed the study. Q. Sun, L. Fang, X. Tang, M. Roth and E. Papakonstantinou performed experiments, and analysed the data. W. Zhai and Q. Sun analysed the data of BALF proteins by KEGG pathway analysis. R. Louis, V. Heinen, F.N. Schleich, D. Stolz and M. Tamm provided the lung tissues, patients' clinical data, and contributed to manuscript preparation and writing. S. Savic contributed lung tissue section for histology. Q. Sun, S. Lu, M. Tamm, D. Stolz and M. Roth had scientific discussion of the study and wrote the paper. All authors read and approved the manuscript.

Conflict of interest: Q. Sun has nothing to disclose. L. Fang has nothing to disclose. M. Roth has nothing to disclose. $\mathrm{X}$. Tang has nothing to disclose. E. Papakonstantinou has nothing to disclose. W. Zhai has nothing to disclose. R. Louis reports grants and personal fees for advisory board work from GSK, AstraZeneca and Novartis, and grants from Chiesi, outside the submitted work. V. Heinen has nothing to disclose. F.N. Schleich has nothing to disclose. S. Lu has nothing to disclose. S. Savic has nothing to disclose. M. Tamm has nothing to disclose. D. Stolz has nothing to disclose. 
Support statement: This work was supported by Swiss National Foundation grant 31003A_176248/1, Swiss Lung Association grant 2017-02_Roth to M. Roth, a Swiss Government Excellence Scholarship to Q. Sun 2014.0405, and National Natural Science Foundation of China grants 31871314 and 31601033 to S. Lu. Funding information for this article has been deposited with the Crossref Funder Registry.

\section{References}

1 Pare PD, Roberts CR, Bai TR, et al. The functional consequences of airway remodelling in asthma. Monaldi Arch Chest Dis 1997; 52: 589-596.

2 Elias JA. Airway remodelling in asthma. Unanswered questions. Am J Respir Crit Care Med 2000; 161: S168-S171.

3 Berair R, Saunders R, Brightling CE. Origins of increased airway smooth muscle mass in asthma. BMC Med 2013; 11: 145.

4 Weitoft M, Andersson C, Andersson-Sjoland A, et al. Controlled and uncontrolled asthma display distinct alveolar tissue matrix compositions. Respir Res 2014; 15: 67.

5 Salem IH, Boulet LP, Biardel S, et al. Long-term effects of bronchial thermoplasty on airway smooth muscle and reticular basement membrane thickness in severe asthma. Ann Am Thorac Soc 2016; 13: 1426-1428.

6 Chakir J, Haj-Salem I, Gras D, et al. Effects of bronchial thermoplasty on airway smooth muscle and collagen deposition in asthma. Ann Am Thorac Soc 2015; 12: 1612-1618.

7 Janssen LJ. Airway smooth muscle as a target in asthma and the beneficial effects of bronchial thermoplasty. J Allergy 2012; 2012: 593784.

8 Loxham M, Davies DE, Blume C. Epithelial function and dysfunction in asthma. Clin Exp Allergy 2014; 44: $1299-1313$.

9 Lambrecht BN, Hammad H. The airway epithelium in asthma. Nat Med 2012; 18: 684-692.

10 Prakash YS, Halayko AJ, Gosens R, et al. An Official American Thoracic Society Research Statement: Current Challenges Facing Research and Therapeutic Advances in Airway Remodelling. Am J Respir Crit Care Med 2017; 195: e4-e19.

11 Boulet LP. Airway remodelling in asthma: update on mechanisms and therapeutic approaches. Curr Opin Pulm Med 2018; 24: 56-62.

12 Pretolani M, Bergqvist A, Thabut G, et al. Effectiveness of bronchial thermoplasty in patients with severe refractory asthma: clinical and histopathologic correlations. J Allergy Clin Immunol 2017; 139: 1176-1185.

13 Denner DR, Doeing DC, Hogarth DK, et al. Airway inflammation after bronchial thermoplasty for severe asthma. Ann Am Thorac Soc 2015; 12: 1302-1309.

14 O'Reilly A, Browne I, Watchorn D, et al. The efficacy and safety of bronchial thermoplasty in severe persistent asthma on extended follow-up. QJM 2018; 111: 155-159.

15 DeVries A, Vercelli D. Epigenetic mechanisms in asthma. Ann Am Thorac Soc 2016; 13: Suppl. 1, S48-S50.

16 Kidd CD, Thompson PJ, Barrett L, et al. Histone modifications and asthma. The interface of the epigenetic and genetic landscapes. Am J Respir Cell Mol Biol 2016; 54: 3-12.

17 Sun Q, Liu L, Wang H, et al. Constitutive high expression of protein arginine methyltransferase 1 in asthmatic airway smooth muscle cells is caused by reduced microRNA-19a expression and leads to enhanced remodelling. J Allergy Clin Immunol 2017; 140: 510-524.

18 Wei H, Mundade R, Lange KC, et al. Protein arginine methylation of non-histone proteins and its role in diseases. Cell Cycle 2014; 13: 32-41.

19 Sun Q, Liu L, Roth M, et al. PRMT1 upregulated by epithelial proinflammatory cytokines participates in COX2 expression in fibroblasts and chronic antigen-induced pulmonary inflammation. J Immunol 2015; 195: 298-306.

20 Sun QZ, Yang XD, Zhong B, et al. Upregulated protein arginine methyltransferase 1 by IL-4 increases eotaxin-1 expression in airway epithelial cells and participates in antigen-induced pulmonary inflammation in rats. J Immunol 2012; 188: 3506-3512.

21 Sun Q, Liu L, Mandal J, et al. PDGF-BB induces PRMT1 expression through ERK1/2 dependent STAT1 activation and regulates remodelling in primary human lung fibroblasts. Cell Signal 2016; 28: 307-315.

22 Liu L, Sun Q, Bao R, et al. Specific regulation of PRMT1 expression by PIAS1 and RKIP in BEAS-2B epithelia cells and HFL-1 fibroblasts in lung inflammation. Sci Rep 2016; 6: 21810.

23 Sun Q, Fang L, Tang X, et al. TGF- $\beta$ upregulated mitochondria mass through the SMAD2/3 $\rightarrow$ C/EBP $\beta \rightarrow$ PRMT1 signal pathway in primary human lung fibroblasts. J Immunol 2019; 202: 37-47.

24 Crosby LM, Waters CM. Epithelial repair mechanisms in the lung. Am J Physiol Lung Cell Mol Physiol 2010; 298 L715-L731.

25 Yan Y, Hanse EA, Stedman K, et al. Transcription factor C/EBP-beta induces tumor-suppressor phosphatase PHLPP2 through repression of the miR-17-92 cluster in differentiating AML cells. Cell Death Differ 2016; 23: $1232-1242$.

26 Girodet PO, Allard B, Thumerel M, et al. Bronchial smooth muscle remodelling in nonsevere asthma. Am J Respir Crit Care Med 2016; 193: 627-633.

27 Girodet PO, Ozier A, Bara I, et al. Airway remodelling in asthma: new mechanisms and potential for pharmacological intervention. Pharmacol Ther 2011; 130: 325-337.

28 Aravamudan B, Kiel A, Freeman M, et al. Cigarette smoke-induced mitochondrial fragmentation and dysfunction in human airway smooth muscle. Am J Physiol Lung Cell Mol Physiol 2014; 306: L840-L854.

29 Kol A, Lichtman AH, Finberg RW, et al. Cutting edge: heat shock protein (HSP) 60 activates the innate immune response: CD14 is an essential receptor for HSP60 activation of mononuclear cells. J Immunol 2000; 164: 13-17.

30 Sell H, Poitou C, Habich C, et al. Heat shock protein 60 in obesity: effect of bariatric surgery and its relation to inflammation and cardiovascular risk. Obesity 2017; 25: 2108-2114.

31 Marker T, Sell H, Zillessen P, et al. Heat shock protein 60 as a mediator of adipose tissue inflammation and insulin resistance. Diabetes 2012; 61: 615-625.

32 Park MJ, Kim DI, Lim SK, et al. Thioredoxin-interacting protein mediates hepatic lipogenesis and inflammation via PRMT1 and PGC-1alpha regulation in vitro and in vivo. J Hepatol 2014; 61: 1151-1157.

33 Shen NY, Ng SY, Toepp SL, et al. Protein arginine methyltransferase expression and activity during myogenesis. Biosci Rep 2018; 38: BSR20171533. 